\title{
Leonardo da Vinci, scienziato*
}

\author{
R. Pucci ${ }^{1,2 \dagger} \odot$, G. G. N. Angilella ${ }^{1,2,3,4,5 \ddagger}$ \\ ${ }^{1}$ Dipartimento di Fisica e Astronomia "Ettore Majorana", Università di Catania, \\ Via S. Sofia, 64, I-95123 Catania, Italy \\ ${ }^{2}$ CNR-IMM, Catania, Italy \\ ${ }^{3}$ INFN, Sez. Catania, Catania, Italy \\ ${ }^{4}$ CNISM, UdR Catania, Italy \\ ${ }^{5}$ Scuola Superiore di Catania, Università di Catania, \\ Via Valdisavoia 9, I-95123 Catania, Italy
}

\section{Riassunto}

Leonardo è stato l'unico scienziato la cui ricerca non solo ha preso spunto da immagini, ma è avvenuta con immagini. Egli ha espresso le sue idee scientifiche non con parole o formule, ma con un linguaggio visivo. Leonardo è questa anomalia. Non si ripeterà nella storia della scienza una simile coincidenza fra scienza ed arte. Egli, inoltre, effettua una continua simbiosi tra diversi campi della scienza. Egli, ad esempio, trasferisce i suoi studi delle onde nell'acqua alle onde dell'aria o i suoi studi nell'ottica alla prospettiva nell'arte o le proprietà delle leve allo studio del moto degli arti. Leonardo è stato definito l'uomo più curioso che sia mai esistito, ed in effetti i suoi interessi spaziano tra tutti i saperi noti alla sua epoca: dalla pittura alla scultura, dalla meccanica all'idraulica, dall'architettura alla geologia, dall'anatomia alla zoologia, dalla fisiologia alla geografia, dalla matematica all'ottica, dalla statica all'astronomia ed alla botanica. In questa conferenza si accenna ad alcune delle più importanti scoperte di Leonardo, sottolineando il carattere di rottura che esse hanno rispetto alle idee dominanti nella scienza del suo tempo.

Parole chiave: Leonardo da Vinci (1452-1519), storia della scienza, epistemologia.

* Science colloquium presentato dal Membro Effettivo Renato Pucci, Professore Emerito nell'Università di Catania, presso il Dipartimento di Fisica e Astronomia 'Ettore Majorana' dell'Università di Catania il 10 aprile 2019.

†renato.pucci@ct.infn.it (Autore corrispondente)

‡giuseppe.angilella@ct.infn.it 


\section{Summary}

\section{Leonardo da Vinci, scientist}

Among all scientists, Leonardo's research was not only inspired by images, but also achieved through images. He expressed his scientific ideas not just via words or formulas, but by means of a visual language. Leonardo is this anomaly. Never in science will such a coincidence between science and art be repeated. Moreover, he merged different fields of science, in a continuous symbiosis. For instance, he transferred his studies on water waves to atmospheric waves, or his studies in optics to perspective in visual arts, or the properties of mechanical levers to the study of the motion of limbs. Leonardo has been defined as the most curious man who has ever existed. Actually, his interests span across all known subjects in his age: from painting to sculpture, from mechanics to hydraulics, from architecture to geology, from anatomy to zoology, from physiology to geography, from mathematics to optics, from statics to astronomy and botanics. In this seminar, some of Leonardo's most important achievements will be touched upon, with an emphasis to their breakthrough character with respect to the scientific dominant ideas in his time.

Keywords: Leonardo da Vinci (1452-1519), history of science, epistemology.

\section{Introduzione}

Il 15 aprile 1452, Leonardo nasce a Vinci (Granducato di Toscana), come figlio naturale di Piero da Vinci, notaio, e di Caterina, figlia di contadini. Il 2 maggio 1519, Leonardo muore nel castello di Cloux, non lontano da Amboise, sulla Loira, residenza di Francesco I, Re di Francia. Ricorre pertanto quest'anno 2019 il $500^{\circ}$ anniversario della morte del celebre scienziato, la cui grandezza tuttavia è spesso associata a caratteristiche del suo multiforme ingegno che punto o poco hanno a che vedere con il suo essere uomo di scienza.

Leonardo (Fig. 1) fu certamente uomo del suo spazio e del suo tempo, toscano per nascita, italiano ante litteram ed anzi europeo per vocazione. Nasce illetterato, diventa quasi erudito da autodidatta, per curiosità. Esplora la suddivisione medievale (quasi: la classificazione) della conoscenza in trivio e quadrivio, e la trascende, valicando confini non solo geografici e politici, ma piuttosto attraversando e anzi fondendo il limite fra scienza ed arte, in questo costituendo egli stesso un punto di transizione, come proveremo ad argomentare nella seguente, breve esposizione.

\section{Brevi note epistemologiche}

Kuhn (1962) ha dimostrato che la storia della scienza si può descrivere come lunghi periodi di scienza normale alternati da brevi periodi di scienza rivoluzionaria. I periodi di scienza rivoluzionaria sono legati a periodi storici di grandi cambiamenti sociali, politici ed economici. Il Rinascimento fu senz'altro uno di 


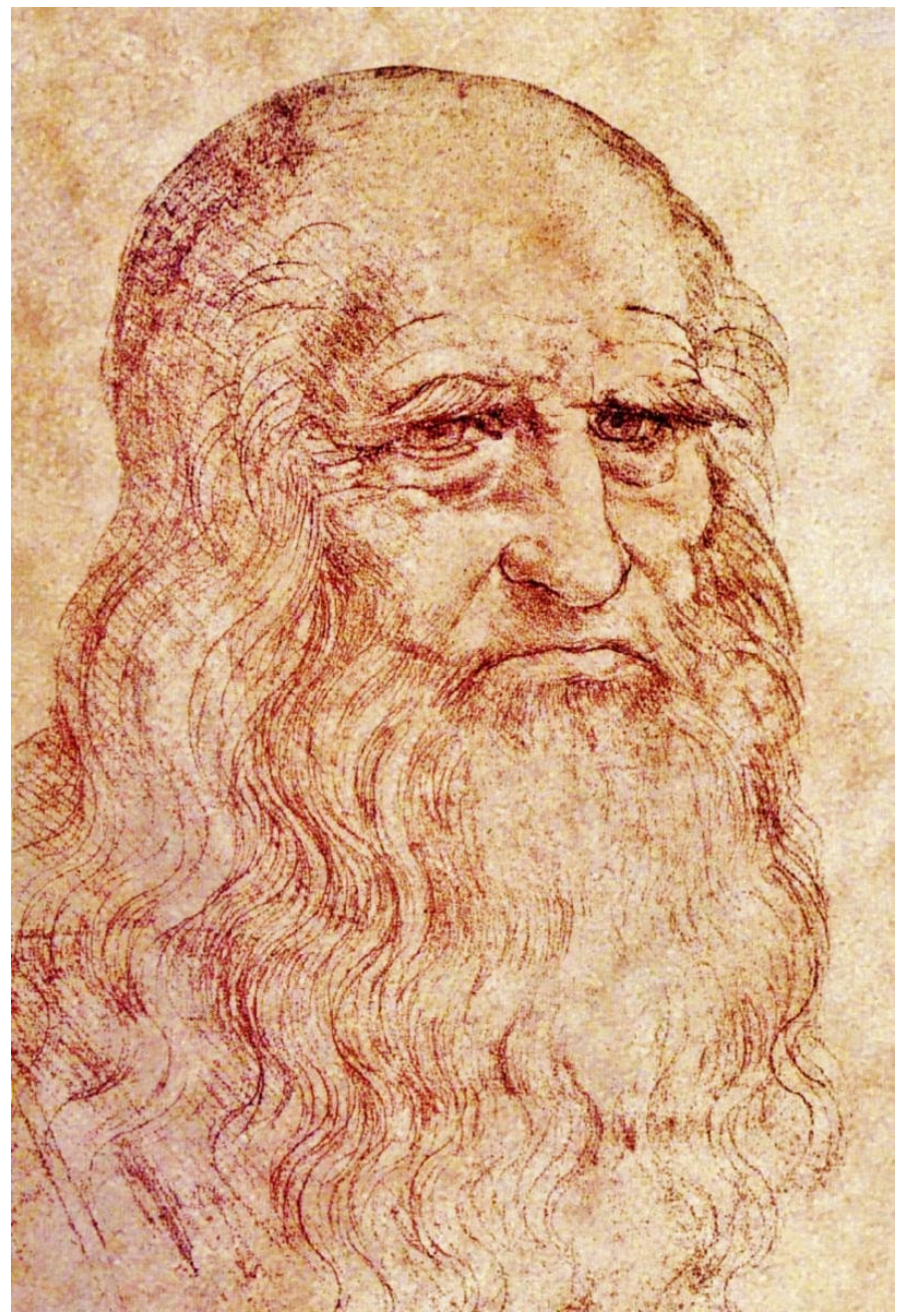

Figura 1: Leonardo da Vinci (1452-1519), Ritratto d'uomo (sanguigna su carta: Biblioteca Reale, Torino). L'opera è quasi universalmente considerata l'autoritratto del genio italiano.

questi periodi e produsse scienziati quali Leonardo, Galileo, Cartesio, e Newton. Nei periodi rivoluzionari, alcuni scienziati, per lo più giovani, osano ribellarsi al sapere comunemente accettato in quel periodo.

Scrive Dyson (2006):

“Non c'è un'unica visione scientifica, come non c'è un'unica visione poetica. La scienza è un unico mosaico di visioni parziali e conflittuali. Ma c'è un elemento comune in queste visioni. L'elemento comune è la ribellione contro le restrizioni imposte dalla cultura dominante."

Rovelli (2011) è ancor più preciso, dicendo che uno scienziato introduce innovazioni rilevanti solo se è capace di contraddire i suoi maestri in qualche aspetto fondamentale (cf. anche Pucci and Angilella, 2017).

Il xv secolo fu un'epoca di grandi sconvolgimenti economici, politici, religiosi e sociali. La caduta di Costantinopoli nel 1453 portò ad una diaspora di intellettuali provenienti da quel mondo, rifugiatisi soprattutto in Italia, e ad una ripresa 


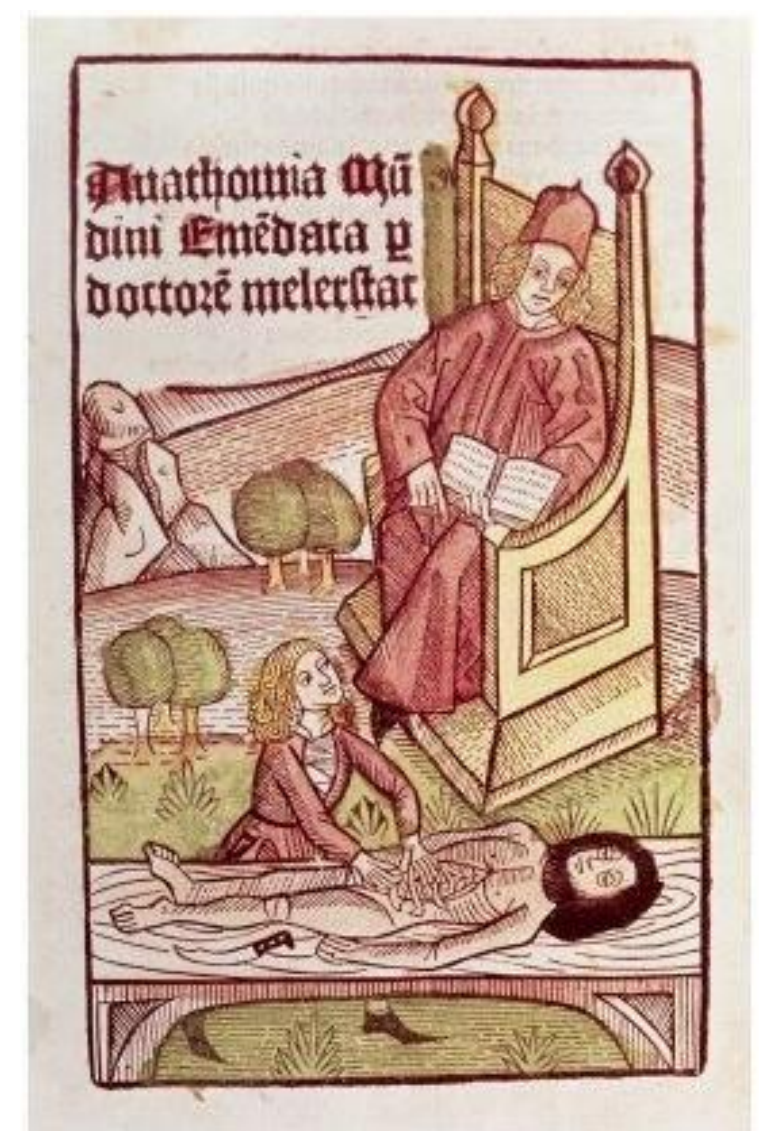

Figura 2: Mondino de' Liuzzi, Lezione di anatomia (frontespizio de Anathomia Mundini, 1493).

degli studi dei testi greco-romani. Leonardo fu uno dei maggiori interpreti di quella rinascita. Di lui scrive il Vasari (1568):

"Grandissimi doni si veggono piovere dagli influssi celesti nei corpi umani molte volte naturalmente, e sopra naturali, talvolta, strabocchevolmente accozzarsi in un corpo solo bellezza, grazie e virtù [...]. Questo lo videro gli uomini in Lionardo da Vinci, nel quale oltra la bellezza del corpo, non lodata mai abbastanza, era la grazia infinita in qualunque sua azione; e tanta e sì fatta poi la virtù, che dovunque l'animo volse nelle cose difficili, con facilità le rendeva assolute. La forza in lui fu molta e congiunta con la destrezza, l'animo e'l valore, sempre regio e magnanimo."

\section{Il metodo scientifico}

La Fig. 2 riproduce una stampa del $x v$ secolo che raffigura, in alto, il professorefilosofo naturale con i testi degli auctores, e in basso il chirurgo, che in virtù del suo sapere manuale, disseziona il corpo. L'ambiente culturale è lo studium, cioè l'università. Un simile rapporto tra teoria e pratica viene riportato in questo periodo anche per le altre discipline. 
Leonardo, soprattutto nel periodo fiorentino (1452-1481), definisce se stesso un uomo senza lettere. Egli infatti non aveva frequentato l'università, ma la bottega del Verrocchio, che era illustre pittore, scultore, restauratore, costruttore di macchine. Leonardo, però, non aveva alcun timore reverenziale in quanto era consapevole di essere discepolo dell'esperienza. Diceva che ogni discorso che non passa dall'esperienza della realtà è vano, perché non nasce da una conoscenza diretta delle cose e non può portare nessuna utilità all'uomo e al suo progresso (Laurenza, 1999).

"Fuggi i precetti di quei teorici che non confermano le loro ragioni con l'esperienza", amava ripetere. Nei suoi ragionamenti non tentava mai di forzare i fatti per farli andare d'accordo con le ipotesi che gli stavano a cuore, e piuttosto lasciava che l'esperienza dei fenomeni gli illuminasse la via. In questo fu un precursore di quel metodo scientifico che sarebbe stato messo a punto solo un secolo dopo dal lavoro di Cartesio, Galileo e Newton.

Ma nel periodo milanese (1482-1499) rivalutò lo studio teorico: studiò appassionatamente il latino e lesse nella versione originale 'classici' come Euclide ed Archimede. Disponeva di una biblioteca di 105 libri. $^{1} \mathrm{Fa}$ amicizia con il famoso matematico Luca Pacioli ed altri 'intellettuali', quali ad esempio Marcantonio della Torre, professore di anatomia all'Università di Pavia. Leonardo ora mostra di essere sensibile al valore della matematica: "La meccanica", scriveva, "è il paradiso delle scienze matematiche, perché con quella si viene al frutto matematico."

Egli, inoltre, alla fine del libro della pittura scrive: "Nissuna umana investigazione si può dimandare vera scienza, se essa non passa per le matematiche dimostrazioni. E se tu dirai che le scienze, che principiano e finiscono nella mente, abbiano verità, questo non si concede, ma si niega per molte ragioni: e primo, che in tali discorsi mentali non accade esperienza, sanza la quale nulla dà di sé certezza."

"Se Leonardo merita un posto di rilievo nella storia della scienza, oltre che in quello dell'arte, lo deve non al fatto che ha fantasticato — senza saperli realizzare- di sommergibili e macchine volanti, ma alla forma visiva in cui ha espresso le sue riflessioni sulla natura. Questo è avvenuto sia attraverso l'utilizzo di disegni e schemi visivi, sia attraverso la creazione di opere d'arte.

La sua ricerca scientifica non solo prende spunto da immagini, ma avviene con immagini. Egli esprime le sue idee scientifiche non con parole, ma con un linguaggio visivo. Leonardo è questa anomalia. Non si ripeterà nella storia della scienza una simile coincidenza fra scienza ed arte." (Klein, 2008)

L'unico, forse, che si potrebbe ricordare è Feynman (1949), che con i suoi diagrammi ha reso visibili i processi elementari d'interazione (cf. Kaiser, 2005). ${ }^{2}$

\footnotetext{
${ }^{1}$ La mostra interattiva Leonardo e i suoi libri: La biblioteca del Genio Universale, a cura di Carlo Vecce, visitabile da giugno a settembre 2019 presso il Museo Galileo di Firenze, ma disponibile online anche oltre tale data, tenta per la prima volta di ricostruire la biblioteca di Leonardo, attraverso le liste manoscritte dei suoi libri.

${ }^{2}$ Cita Rovelli (2015) dall'autobiografia di Nabokov (1966), che oltre ad essere letterato fu anche entomologo, dunque uomo di scienza: "Ho scoperto nella natura un dilettarsi senza utilità che ho cercato nell'arte. Entrambe sono una forma di magia, entrambe un gioco di intricato incanto e inganno." E aggiunge Rovelli (2015): "C'è qui ben più che la sola capacità di osservare $i$ dettagli
} 


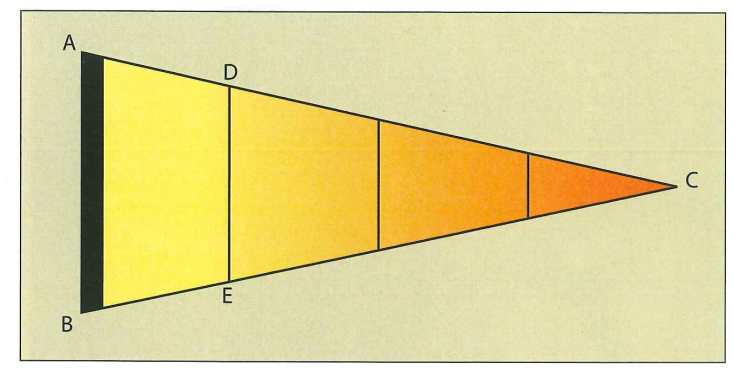

Figura 3: La legge della piramide.

Egli, inoltre, effettua una continua simbiosi tra diversi campi della scienza. Egli, ad esempio, trasferisce i suoi studi delle onde nell'acqua alle onde dell'aria o i suoi studi nell'ottica alla prospettiva nell'arte, o le proprietà delle leve allo studio del moto degli arti.

Leonardo è stato definito l'uomo più curioso che sia mai esistito, ed in effetti i suoi interessi spaziano su tutti i saperi noti alla sua epoca: dalla pittura alla scultura, dalla meccanica all'idraulica, dalla architettura alla geologia, dall'anatomia all'astronomia, dalla fisiologia alla geografia, dalla matematica all'ottica, dalla statica alla zoologia ed alla botanica. La sua dedizione fu straordinaria. "Egli riuscì a continuare ad occuparsi di un problema per decenni, anche quando sembrava che non ci fossero prospettive di pervenire ad una soluzione, a causa della insufficiente acutezza della propria mente. Da dove veniva questo 'ostinato rigore', questa capacità di soffrire per un compito che si era autoimposto? Facendo riferimento al suo maestro Verrocchio, Leonardo ebbe a dire che la cosa più importante che un maestro possa dare ad un allievo non è né l'esperienza né il sapere, bensì l'entusiasmo." (Klein, 2008)

\section{Ottica: la legge della piramide}

Leonardo lavorò per anni per fondare le regole della prospettiva sulle leggi dell'ottica. Seguendo l'Alberti, chiamò la regola secondo la quale gli oggetti crescono o si rimpiccioliscono apparentemente al variare delle distanze legge della piramide.

Se si disegnano i raggi di luce che vanno dalla periferia dell'oggetto all'occhio, si forma una piramide (Fig. 3). Egli cioè comprese la legge geometrica generale che si cela dietro questo stato di cose: l'estensione apparente di un oggetto

con ossessionata attenzione. C'è la capacità di vedere la bellezza." Un altro scienziato, stavolta fisico matematico, che ebbe a dichiarare di lasciarsi persino guidare dalla bellezza delle formule verso la verità del loro significato. Noi crediamo che le simmetrie costituiscano la struttura delle leggi della fisica e che la ricerca delle simmetrie (e della rottura delle simmetrie, cf. Anderson, 1972) sia un potente metodo di indagine scientifica (Pucci, 2017), ma non bisogna correre il rischio di trascurare o sottovalutare l'indagine sperimentale, che è l'unica in grado di poter falsificare le nostre teorie (Popper, 1935, 1963). La scienza acquisirebbe altrimenti prerogative tipiche delle religioni, con conseguenti eresie, scismi etc. Wilczek (2015), Premio Nobel per la Fisica nel 2004, arriva addirittura ad asserire: "In beauty we trust." 


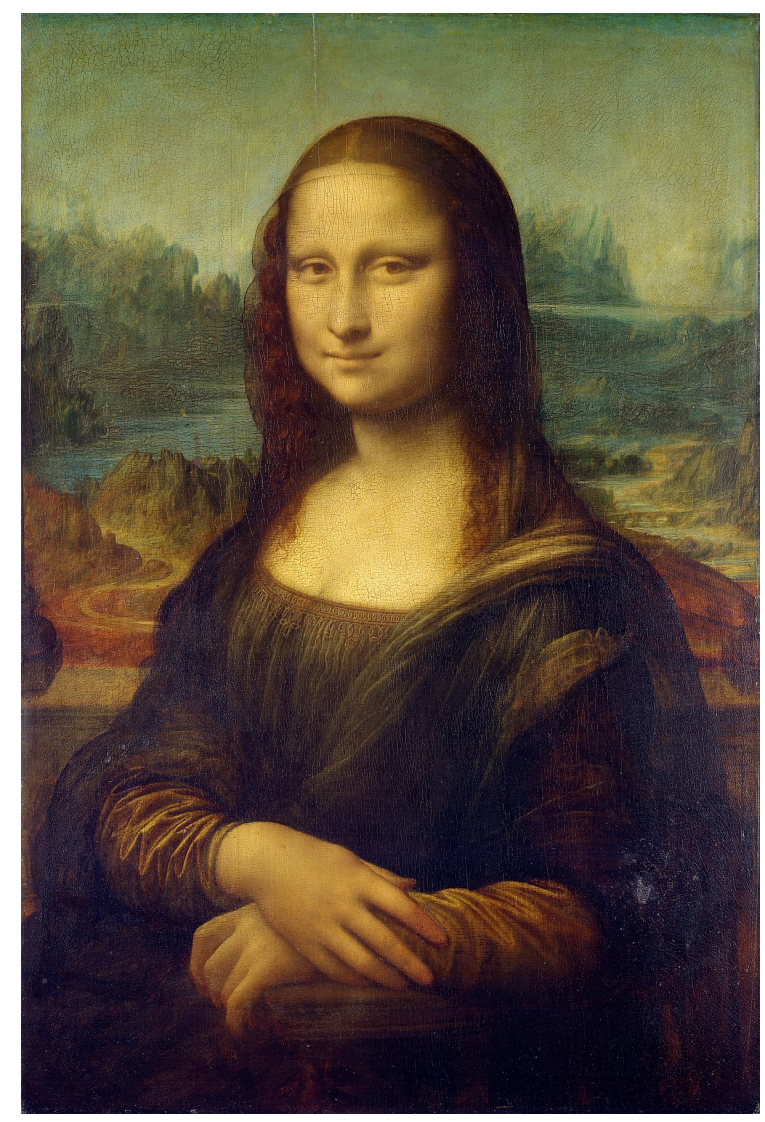

Figura 4: Leonardo, Monna Lisa o La Gioconda (ca. 1503, Louvre, Parigi).

cresce o diminuisce uniformemente in relazione alla distanza dall'osservatore (Kemp, 1982).

Leonardo estese questo effetto anche ai colori: "Quello che tu vuoi che sia cinque volte più lontano, fallo cinque volte più azzurro" (Codice Leicester). Le montagne dietro l'immagine della Gioconda ci appaiono lontane a causa del loro colore e dei contorni sfumati (Fig. 4). Il gioco studiato ed attento di luci ed ombre fa quasi emergere il viso di Monna Lisa dal quadro. Leonardo inoltre affermò che la luce variasse, essendo "vaporata in minutissimi e sensibili atomi."

\section{$5 \quad$ Le leggi del moto}

Per Aristotele movimento significa non solo spostamento da un luogo ad un altro, ma anche trasformazione dello stato e della forma di un corpo in un'altra o, come anche viene definita, corruzione. Per quanto riguarda il moto locale, Aristotele distingue tra moti naturali e moti violenti. Lo spazio non è omogeneo ed isotropo, ma vi sono luoghi naturali. Ad esempio, il luogo naturale dei corpi pesanti è il centro della Terra, mentre quello del fuoco è il cielo. I moti naturali sono quelli che portano un corpo verso il suo luogo naturale (ad esempio, una pietra che cade) e può avvenire solo in linea retta o circolare. I moti violenti, invece, allontanano dal luogo naturale, richiedendo l'applicazione continua di una 
forza e avvengono con orbite di forma qualunque (vedi il moto di un proiettile). I moti violenti possono esistere solo al di sotto dell'orbita lunare. Aristotele, inoltre, doveva ricorrere alla teoria dell'antiperistasi per spiegare il moto violento di un mobile, ad esempio una freccia, quando questa veniva scagliata. Questa teoria prevedeva la partecipazione attiva dell'aria, che passando dietro al mobile lo spingeva facendo sì che esso continuasse a muoversi anche lontano dalla causa che aveva generato il suo movimento. Nel Quattrocento, tale teoria fu aspramente criticata da alcuni studiosi fautori della teoria dell'impetus. Tra questi, vanno menzionati Filopono, Ockam, Burtley, Nemorario, Buridano (rettore dell'Università di Parigi nel 1327), Alberto di Sassonia e Nicola Oresme (rettore del Collegio di Navarra nel 1362 e vescovo di Lisieux nel 1377) (Maccarrone, 2019).

La teoria dell'impeto rimase però minoritaria negli atenei. Secondo questa teoria bisogna supporre che il motore imprima all'oggetto mosso (chiamato mobile) uno slancio, appunto l'impetus, il quale era incorporato nell'oggetto stesso e risultava essere la causa del moto. Secondo Buridano, "tale slancio è tanto più intenso quanto maggiori sono la quantità di materia e la velocità dell'oggetto" (Buridanus, 1509, II, xII). Inoltre, a causa della resistenza dell'aria e del peso del corpo, l'impetus decresce col passare del tempo. Quando l'impetus è esaurito il moto cessa! Leonardo aderì alla teoria di Aristotele del moto come metamorfosi dei corpi, ma per quanto riguarda il moto locale sposò la teoria dell'impetus, rigettando decisamente l'antiperistasi e facendo ricorso al moto dei fluidi.

Nel Codice Leicester $(29 \mathrm{v})$ osserva che una pallottola sparata da uno scoppietto in un "otre pieno d'acqua" continua a procedere nell'acqua anche se quest'ultima ha separato la pallottola dall'aria. Ha anche un'ancor più sottile motivazione contro l'antiperistasi: quando l'aria passa dietro il corpo in movimento, per riempire il vuoto, percuote contro l'aria che il mobile trascina con sé, ma, prosegue Leonardo, "quando due cose si percuotono e' nasce il moto refresso di ciascuna li quali si convertono in oppositi moti revertiginosi." (Ms $\mathrm{G}, f .85 \mathrm{v}$ ). L'aria cioè si richiude su se stessa, formando vortici in cui si esaurisce la sua energia dinamica senza potere in tal modo spingere il corpo. Egli suggerisce esempi di tipo "visivo, quale il movimento vorticoso della polvere e dell'aria sollevate da un cavallo in corsa" (Ms E, f. 80r). Negato quindi all'aria il ruolo che gli attribuiva l'antiperistasi, Leonardo attribuisce ad essa un ruolo fondamentale in quanto l'impeto "non ha lunga permanenza, perché lo strepito generato dal moto [...] del mobile ci manifesta esso mobile trovare resistenza nell'aria da lui penetrata" (Ms G, $f .72 v$ ).

Si deve tener conto quindi anche della densità dell'aria attraversata dai mobili.

“... non si può dare scienza, se prima non si dà la quantità della condensazione dell'aria percossa da qualunque mobile. La qual condensazione sarà di maggiore $\mathrm{o}$ menore densità, secondo la maggiore $\mathrm{o}$ minore velocità che ha in sé il mobile che lo prieme ..." (Ms E, f. 28v).

Questa profonda consapevolezza dell'importanza dell'aria gli impedí, forse, di arrivare al principio d'inerzia. Egli scrive: "Ogni moto attende al suo mantenimento, 


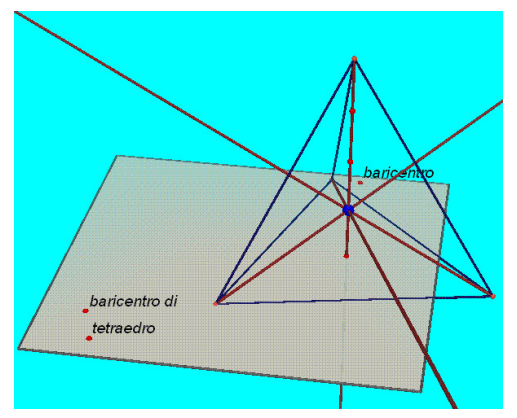

Figura 5: Baricentro di un tetraedro.

ovvero ogni corpo mosso sempre si move in mentre che la impressione che la potentia del suo motore in lui si riserva," ma poi aggiunge: "addunque il moto di tale alia riservando nel suo fine ancora parte della predetta impressione, non potendo per sè seguitare il già principiato che l'impeto della mossa aria è consumato." (Aspettando Galilei ...). Anche per quanto riguarda il principio di azione e reazione Leonardo intuì la sua importanza in alcuni casi particolari, ma non arrivò ed una formulazione generale, che è dovuta a Newton.

Nel Codice Atlantico si legge:

"In quanto al moto dell'acqua tanto fia a movere il remo contro all'acqua immobile, quanto a movere l'acqua contro il remo immobile.

Tanta forza si fa colla cosa in contro all'aria quanto l'aria contro alla cosa.

Tanto fa il moto dell'aria contro alla cosa ferma, quanto il moto del mobile contro all'aria immobile."

Queste affermazioni potrebbero dimostrare che Leonardo intuì la relatività del moto.

\section{Statica}

Il risultato di maggior rilievo in questo campo fu probabilmente lo studio dei centri di gravità di figure piane e solide. Così come Archimede aveva trovato il centro di gravità (chiamato pure centro di massa o baricentro) di un triangolo, Leonardo scoprì il centro di gravità di un tetraedro e di qualsivoglia piramide (Fig. 5). Egli notò, ad esempio, che le linee congiungenti i vertici di un tetraedro con i centri di gravità delle facce opposte passano per uno stesso punto, il centro di gravità del tetraedro. Ampliò il concetto di 'momento' di una forza rispetto ad un punto e formulò in due casi particolari il 'teorema di composizione dei momenti', ed applicò tale risultato a problemi di composizione e scomposizione delle forze. 

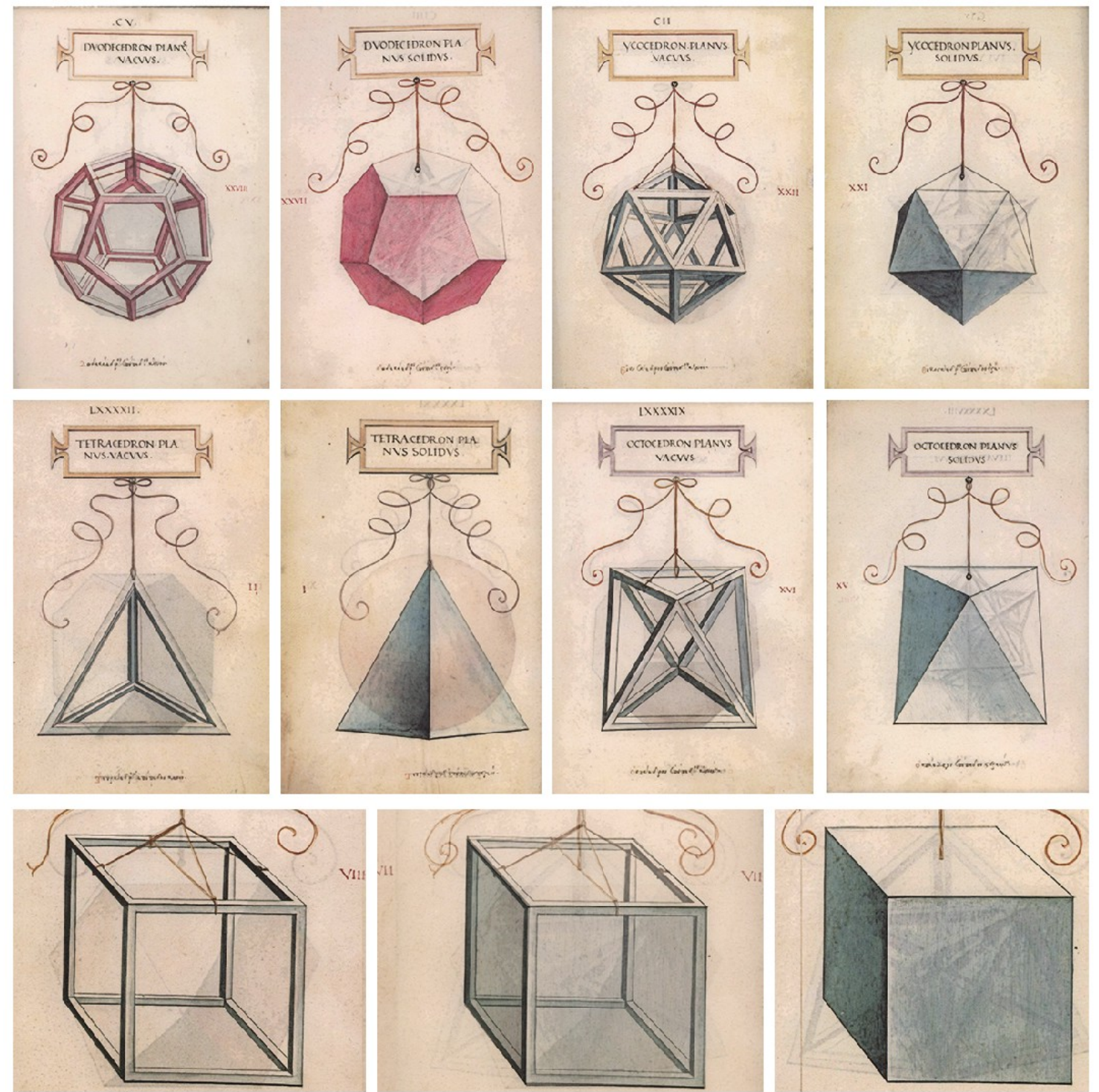

Figura 6: Poliedri regolari nel De divina proportione di Pacioli (1497): incisioni di Leonardo.

\subsection{I solidi}

Leonardo prediligeva la geometria all'algebra, in quanto la geometria necessita di essere visualizzata. La sua lunga e proficua collaborazione con il grande matematico dell'epoca, Luca Pacioli (1447-1517), lo portò a disegnare le figure geometriche su cui si basano le tavole del De divina proportione (Fig. 6). Un esempio sono le tavole con i cinque corpi regolari, i poliedri di origine platonica, simbolo di perfezione ed armonia, che rappresentano la forma degli atomi dei cinque elementi della materia: la piramide per il fuoco, il cubo per la terra, l'ottaedro per l'aria, l'icosaedro per l'acqua, il dodecaedro per il cielo o quintessenza. Ogni poliedro è rappresentato sia in forma piena (solidus) che in trasparenza, come in una sorta di anatomia geometrica (vacuum).

Pietro della Novellara scrisse che nel 1501 Leonardo era così preso dalla geo- 

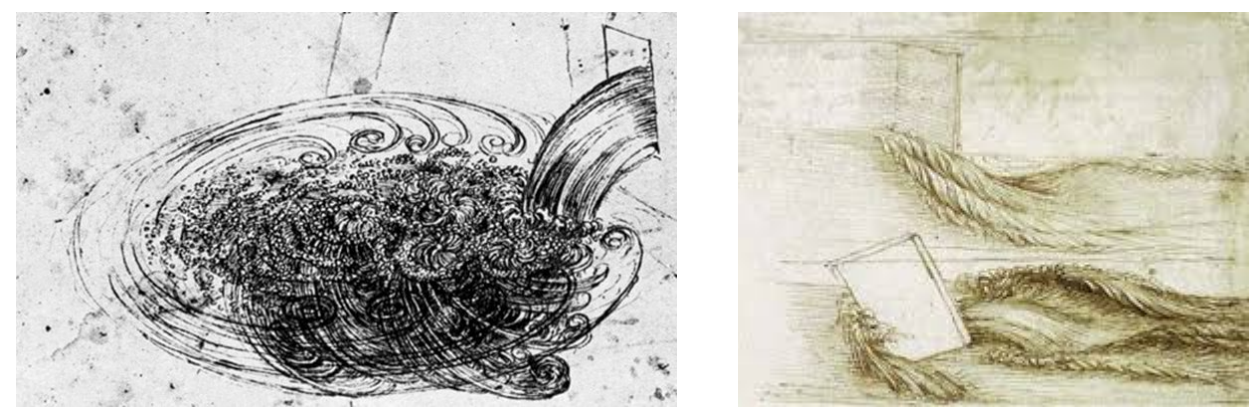

Figura 7: Caduta d'acqua (sinistra) e studi di 'obbietti' nella corrente (destra) (RC, rl 12660v).

metria da essere "impazientissimo al pennello". In effetti egli crede che la struttura del mondo naturale è il dominio di una "necessità e regola eterna" di natura matematica.

\subsection{L'acqua}

Mentre ciò che caratterizza la 'divina' proporzionalità delle scienze esatte, ed in particolare la geometria, è la loro 'invariabilità', ciò che denota il continuo movimento dell'acqua è l'alterazione del letto e degli argini dei fiumi.

"Nel Codice Leicester, pur nel caos dei disegni riportati nei fogli, il motivo dominante è l'acqua. In una figura si vede un getto d'acqua che erompe a dirotto da un'apertura rettangolare in un muro e si getta in un bacino. Il getto è rappresentato solo da un fascio di linee curve che cadono verso il basso. Là dove queste linee incontrano la superficie ferma, si forma una mobile schiuma. L'intero bacino è in sommovimento. L'osservatore intuisce che $i$ vortici alla superficie rappresentano solo la minima parte dei movimenti dell'acqua del bacino. Leonardo cerca di "spiegare visivamente" da dove derivi l'ordine dei vortici d'acqua e pone il primo problema di idrodinamica" (Klein, 2008).

Leonardo si rende conto della complessità del problema e cerca di semplificare lo studio del moto dell'acqua, introducendo il principio di continuità: "poiché in ogni grado della lunghezza del fiume, con egual tempo passa egual quantità d'acqua; se nella larghezza del fiume l'acqua è più veloce da una parte, allora dall'altra parte essa sarà più tarda proporzionalmente." La legge di continuità è anche spiegata con l'esempio di un gruppo di marciatori costretto a passare per due strettoie di diversa larghezza.

Leonardo si dedicò alla ricerca di altre leggi della natura: tentò di spiegare il segreto della tensione superficiale e, secondo Duhem (famoso storico della scienza), intuì il principio di Pascal. Enunciò inoltre le leggi della pressione:

"Se una botte ha in sé il vino alto 4 braccia e gitta il vino lontano da sé 4 braccia, quando il vino sarà nel calare disceso all'altezza di 2 braccia della botte, gitterà ella il vino per la medesima cannella ancora a 2 braccia? Cioè se il calo e l'empito del gittare della cannella diminuisce con eguale proporzione o no." (Biblioteca Nacional, Madrid) 
Se si traduce empito con il concetto moderno di energia, Leonardo è pervenuto al risultato corretto: l'energia dell'acqua diminuisce in modo uniforme all'aumentare dell'altezza del foro d'uscita. Lo studio della gittata dell'acqua dai fori praticati a diversa altezza in un recipiente gli servì anche per prevedere la gittata dei proiettili dei cannoni (esempio del suo uso di semplificazioni e analogie).

Leonardo non solo considerò, infatti, l'aspetto teorico della scienza delle acque, ma anche i suoi giovamenti. Considerò gli "irreparabili e dannosi furori" che l'acqua ha sugli "obbietti", cioè sugli ostacoli (Fig. 7, a sinistra) e suggerì la forma che i piloni dei ponti devono avere per opporre meno resistenza all'acqua (Fig. 7, a destra). Studiò in dettaglio la forma ed il meccanismo di funzionamento dei portoni delle chiuse elaborando un modello efficace per il sollevamento delle imbarcazioni.

\subsection{Il principio di Pascal}

"Se un vaso pieno d'acqua, chiuso da ogni parte, ha due aperture, una centupla dell'altra, mettendo ad ognuna un pistone adeguato, un uomo che spinge il piccolo pistone eguaglierà la forza di cento uomini, che spingeranno quello che è cento volte più grande, e che sarà novantanove volte superiore al primo.

In qualunque proporzione fossero queste aperture, se le forze che agiranno sui pistoni sono come le apertura, esse saranno in equilibrio. Da ciò appare che un vaso pieno d'acqua è un nuovo principio meccanico, e una nuova macchina per moltiplicare le forze quanto si vorrà, poiché un uomo con questo mezzo potrà sollevare qualunque peso che gli si presenterà" Canguilhem (B. Pascal, 1923-1962, in 1968).

\subsection{Sui canali e le carte idrografiche}

I disegni per le costruzioni di canali fluviali furono uno degli impegni maggiori dell'attività di Leonardo. L'energia dell'acqua serviva per le industrie tessili, le officine produttrici di armi, per i mulini necessari per la macinazione dei cereali. I contadini che vivevano intorno a Milano traevano i loro mezzi di sussistenza dal fatto che l'acqua rendeva fertile il suolo, permettendo la coltivazione del riso e del gelso (indispensabile per l'allevamento dei bachi da seta). Pensò di aumentare il trasporto fluviale a Milano e di congiungere Firenze al mare. Concepì una città ideale disposta su tre livelli: uno per i pedoni, uno per i carri, ed uno per l'acqua che doveva portar via scorie ed escrementi. Lavorò anche, sia per scopi pacifici che bellici, alla produzione di carte idrografiche:

"Il corpo della terra, a similitudine de' corpi de li animali, è tessuto di ramificazione di vene, le quali son tutte insieme congiunte, e son costituite a nutrimeto e vivificazione d'essa terra e de' sua creati." $(\mathrm{CL}, f .33 v)$ 


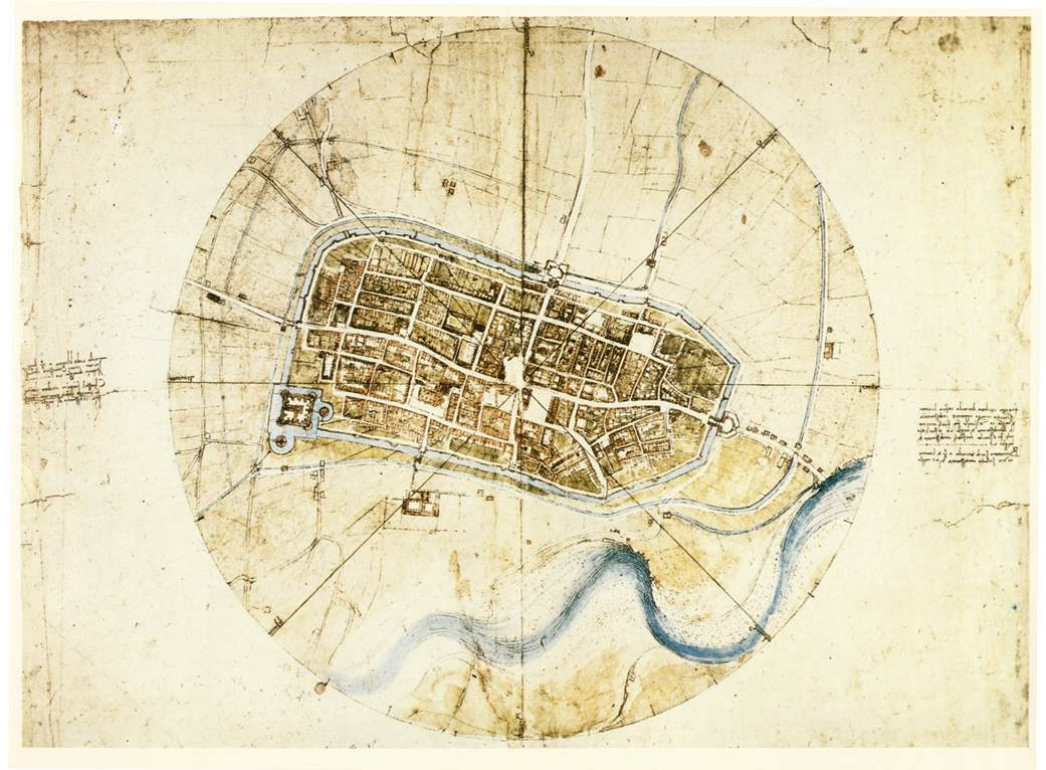

Figura 8: Leonardo, Pianta della città di Imola (1502; The Royal Collection, Windsor).

Splendida anche la mappa 'tridimensionale' di Imola (Fig. 8), che segnò l'inizio della cartografia rinascimentale.

\section{L'uomo come immagine del mondo: microcosmo e macroco- smo in Leonardo}

\subsection{Visione del mondo: la geologia secondo Leonardo}

L'immagine della Terra che disegna Leonardo è quella di una Terra con una storia fatta di un inizio, una fine e, nel mezzo, incessanti cambiamenti. L'immagine di un corpo che, come quello umano, subisce processi di generazione e di corruzione, e che, come questo, va studiato nelle sue parti solide e liquide: terre, rocce, pietre e acqua.

Questa visione storica e anatomica della Terra era allora abbastanza innovativa. Per secoli era prevalsa l'idea di una Terra senza inizio né fine, con piccoli cambiamenti superficiali. Nella religione ebraico-cristiana si ammette che la Terra abbia avuto un inizio, ma per lungo tempo si è ritenuto che essa, tranne il diluvio, non abbia subito cambiamenti. Come al solito, Leonardo si affida all'osservazione e studia: il processo di stratificazione delle rocce, il modellamento della crosta terrestre per effetto dell'acqua (sia di mare che dei fiumi), la diversa natura (argillosa, sabbiosa, ...) degli strati che costituiscono le montagne, i fossili. Per quanto riguarda i fossili, non condivise le ipotesi più diffuse ai suoi tempi, e cioè che fossero dovuti al Diluvio Universale o ad influssi astrali.

Della stoltizia e semplicità di quelli che vogliono che tali animali fossi in alti lochi, distanti dai mari, portati dal diluvio. Come altra setta 

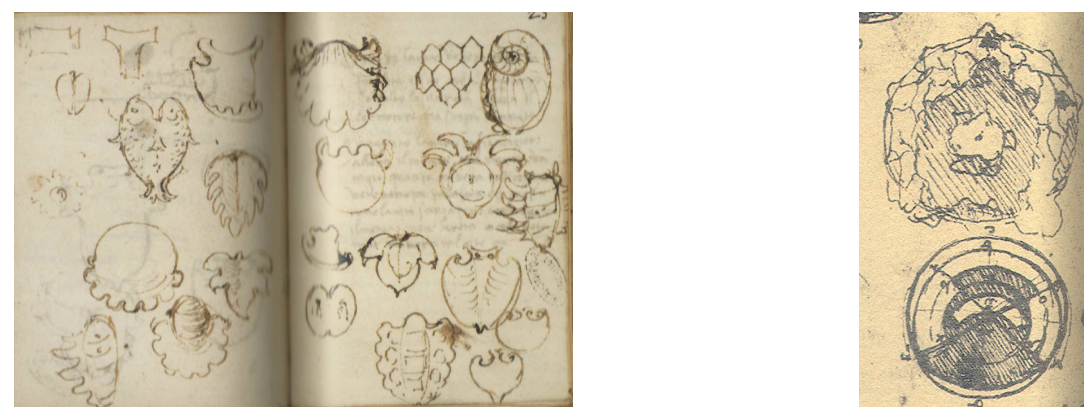

Figura 9: A sinistra: disegni di fossili; il reticolo esagonale in alto a destra, descritto come 'motivo decorativo per architettura' in Galluzzi (2018), è stato più recentemente interpretato come la prima osservazione delle celle realizzate dal fossile Paleodictyon (Barras, 2012) (Ms I, ff. 24v-25r); a destra: rappresentazioni della Terra $(\mathrm{CL}, f$. 36r). Lo schizzo in alto mostra montagne estese anche verso l'emisfero meridionale, mentre lo schizzo in alto dispone le terre emerse prevalentemente nell'emisfero settentrionale.

d'ignoranti affermano la natura o i celi averli in tali lochi creati, per influssi celesti $[\ldots]$ (CL, f. 10r).

Leonardo, invece, crede che i fossili siano una dimostrazione che quelle terre fossero state ricoperte dalle acque. Le conchiglie fossili, come quelle dei corrispondenti animali viventi, mostrano chiaramente gli strati di crescita, analoghi agli strati visibili nei tronchi tagliati dagli alberi. L'intuizione innovativa di Leonardo è consistita nell'aver osservato che gli strati fossili dovevano essersi formati in tempi differenti, permettendo di riconoscere diversi cicli degli anni: su tale concetto si baserà in seguito la distinzione tra ere geologiche. Secondo un recente articolo apparso sulla rivista Nature (Barras, 2012), uno schizzo di Leonardo contenuta nel Ms I di Parigi, raffigurante una struttura esagonale a nido d'ape, potrebbe costituire la prima osservazione registrata di celle realizzate dal fossile Paleodictyon, con implicazioni sulla loro datazione (Fig. 9, a sinistra). Nella Fig. 9 (a destra), Leonardo illustra l'intuizione che il baricentro della Terra non coincidesse con il suo centro, a causa della non uniforme distribuzione delle montagne.

\subsection{Visione dell'uomo: anatomia}

Leonardo dissezionò numerosissimi cadaveri, soprattutto nello 'spedale' Santa Maria Nuova, a Firenze. I fili conduttori delle sue indagini furono due: l'uomo è una macchina, e l'analisi comparata tra uomini vecchi e giovani, tra uomini ed animali, e tra corpo umano e Terra. Il confronto tra il corpo dell'uomo e quello della Terra è confronto tra microcosmo e macrocosmo. Studiare il corso dei fiumi è come studiare il decorso del sangue nei vasi del corpo umano. Alla domanda 'che cos'è la forza' risponde con una 'definizione di forza e movimento negli animali.' Inoltre, i movimenti del corpo umano e animale sono studiati come fossero leve e controleve. Le sue misurazioni spaziano da quelle relative alle 

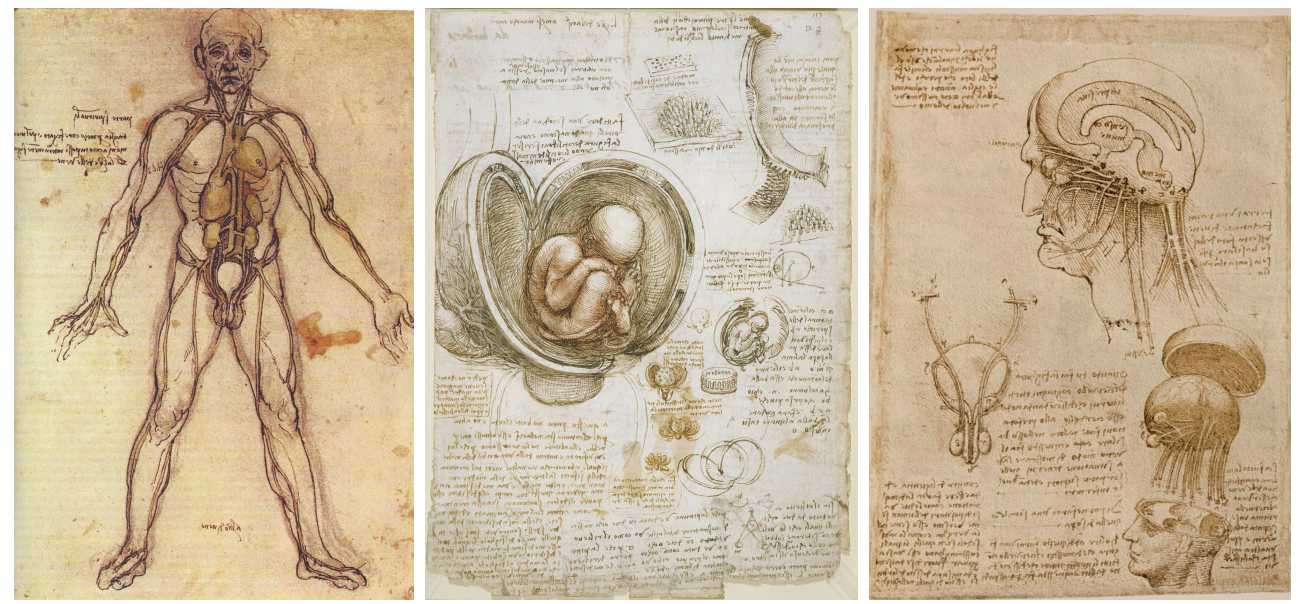

Figura 10: Leonardo, disegni anatomici: albero delle vene (RC, f. 912597r), studio di feto nell'utero (RC, f. 19102r), struttura del cranio e fisiologia del cervello (Weimar, Museo del Castello, inv. KK 6287).

parti dure ed ai loro rapporti (ad esempio cranio, colonna vertebrale, ossa delle gambe etc), a quelle relative alle parti molli. Per queste ultime dovette inventare nuove tecniche di analisi. Ad esempio, fece cuocere l'occhio in albume di uova di gallina, per poterlo poi sezionare.

Leonardo sezionò la cavità del cranio iniettandovi con una siringa della cera fusa. In questo modo poteva ottenerne un calco, a condizione di introdurre 'canne sottili' negli 'sfiatatoi' (buchi praticati nel cranio), "acciò che l'aria che è in essi ventriculi possa spirare e dar loco alla cera innelli ventriculi."

Dice Klein (2008): "Egli ci mostrò le nostre parti molli in modo così plastico da farci provare la tentazione di infilare le dita nell'aorta del cuore da lui disegnato. Egli studiò in gran dettaglio anche l'apparato respiratorio, l'apparato muscolare, quello riproduttivo e quello digerente; sempre proteso a stabilire di ogni organo 'l'uso, l'uffizio e il giovamento'" (Klein, 2008). "I suoi disegni anatomici rappresentano il primo materiale iconografico scientificamente elaborato e aprono la serie dei validi e coraggiosi tentativi di disancoramento dell'anatomia umana dalle concezioni allora imperanti" (Treccani, 2015).

\subsubsection{L'uomo vitruviano}

Leonardo perseguì un'incessante ricerca delle simmetrie in tutti i suoi campi d'indagine: dall'architettura al corpo umano. Nel famoso disegno dell'Uomo Vitruviano (Fig. 11) rappresenta le proporzioni del corpo umano secondo Vitruvio e scrive: "Vitruvio architetto mette nella sua opera d'architettura che le misure dell'omo sono dalla natura distribuite in questo modo ..." Segue una dettagliata analisi dei rapporti tra le varie parti del corpo. A differenza, però, di molti autori suoi contemporanei, egli non iscrive il corpo umano solo in un cerchio, ma lo sdoppia, facendo vedere che è inscrivibile in entrambe le figure geometriche 'perfette': il cerchio ed il quadrato. Viene cioè introdotta la possibilità di due scomposizioni della figura simmetrica e unitaria del corpo umano: homo ad circulum e homo 


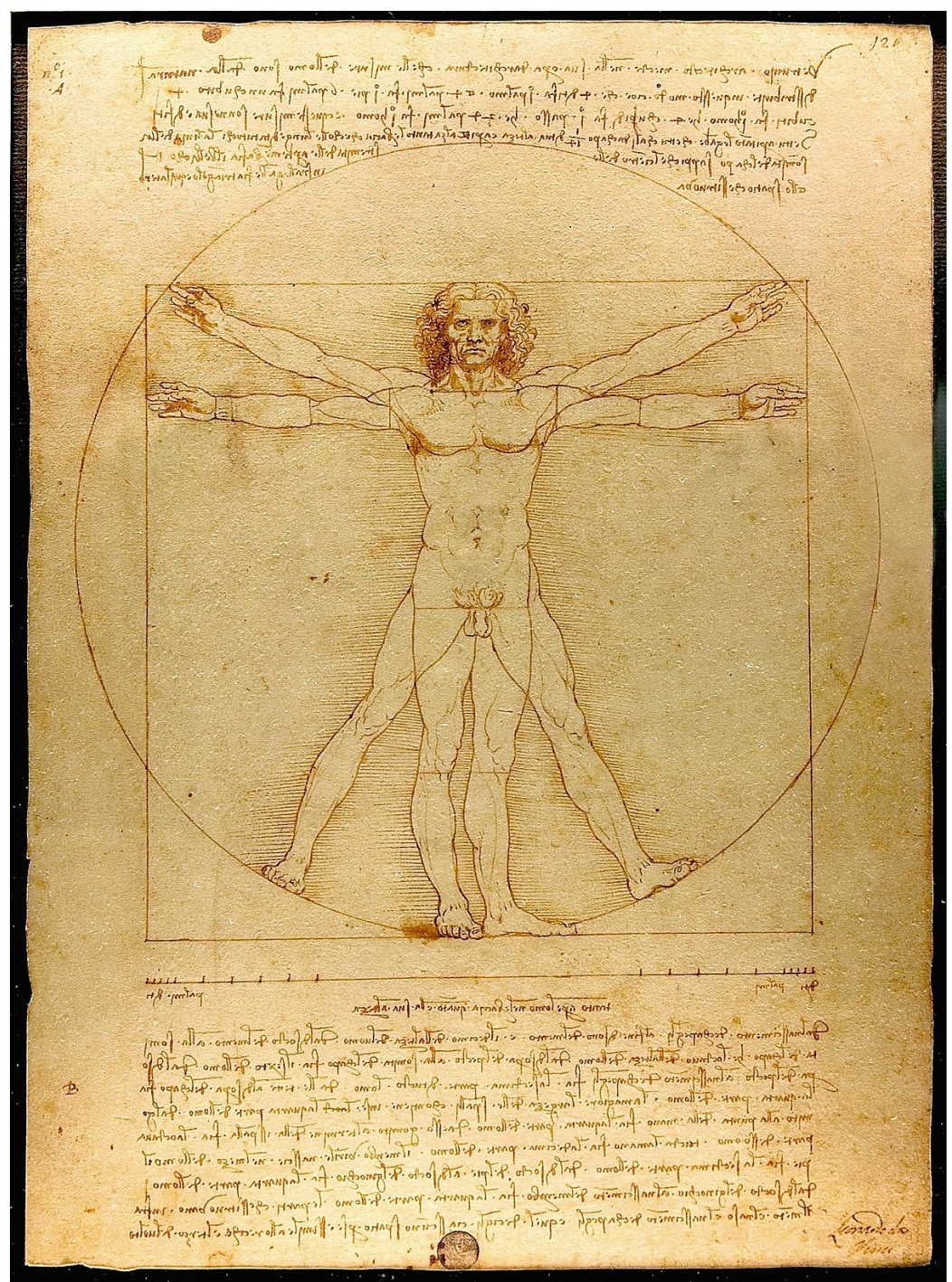

Figura 11: Leonardo, L'uomo vitruviano (Galleria dell'Accademia, Venezia).

ad quadratum. Questo atteggiamento analitico di scomposizione del tutto nelle sue parti viene compensato da una tensione opposta a ricucire le parti nel tutto. Bisogna consultare Pedretti (2008) per avere un'idea di come sia stato sfruttato l'uomo vitruviano di Leonardo; preso come antesignano dei cartoni animati all'ingresso di Disneyland o effigiato sulla tuta degli astronauti statunitensi.

\section{Il sogno del volo}

Nell'antichità la leggenda di Icaro rappresentava l'uomo che voleva volare e raggiungere il cielo, e che veniva giustamente punito per la sua arroganza (Fig. 12). Leonardo parte da un presupposto completamente diverso: l'uomo è simile agli animali e quindi deve poter andare sott'acqua come i pesci (vedi l'invenzione dello scafandro da palombaro) e deve poter volare come gli uccelli. Soprattutto all'esplorazione di questa possibilità egli dedicò grande passione (vedasi il Co- 


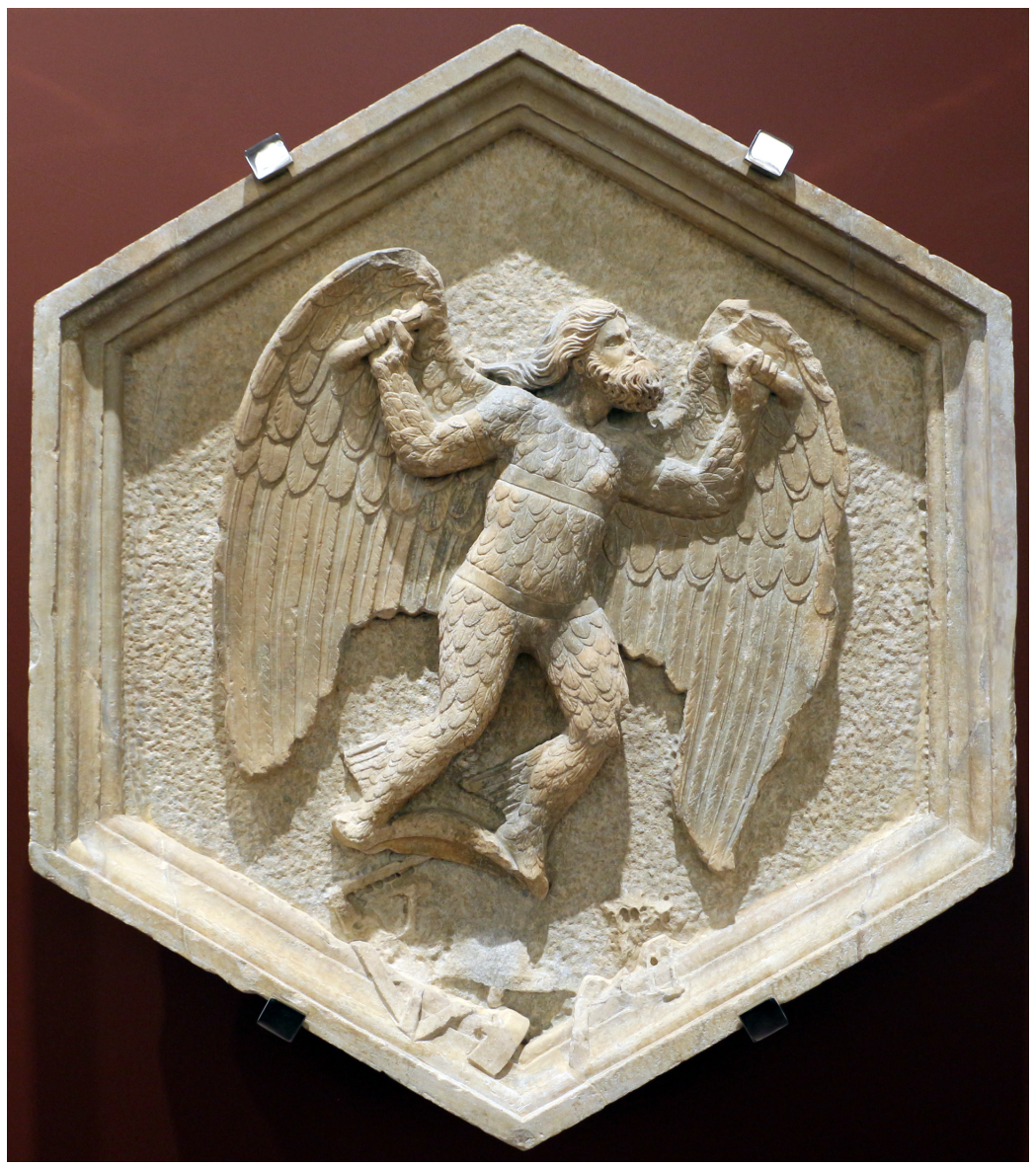

Figura 12: Andrea Pisano (1290-1348), formella alla base del Campanile di Giotto (Firenze).

dice sul volo degli uccelli, CV). Per questa grande sfida, Leonardo osservò il volo planato dei rapaci per poter capire il meccanismo su cui si fonda; contò il numero di colpi d'ala con cui i falchi spiccano il volo; cercò di capire come i rondoni possano raggiungere delle notevoli velocità; disegnò le nuvole e osservò in esse gli inizi dei venti ascendenti e discendenti; studiò l'interazione dei venti con le ali degli uccelli; si fece guidare dall'imitazione delle ali delle aquile e delle poiane per costruire ali utilizzabili dall'uomo.

Probabilmente fece delle prove sul Monte Ceceri, vicino Firenze: "Piglierà il primo volo il grande uccello sopra del dosso del suo magno Cecero, empiendo l'universo di stupore, empiendo di sua fama tutte le scritture e gloria eterna al loco dove nacque." Egli, evidentemente, aspirava ad una fama eterna come costruttore del "grande uccello". In questo sogno voleva farsi guidare dalla natura: "Lo ingegno umano, in invenzioni varie [...], mai esso troverà invenzione più bella né più brieve della natura, perché nelle sue invenzioni nulla manca e nulla è superfluo." Ma fu forse questa scrupolosa imitazione del volo degli uccelli che gli impedì di raggiungere il risultato finale. "In che modo vola l'uccello? La risposta sembra ovvia, ma è sbagliata: battendo le ali. Egli pensava che l'aria si addensasse sotto l'uccello e che quindi il suo corpo venisse spinto da questa verso l'alto" (Klein, 2008). Di conseguenza dotò quasi 
tutte le sue ali di meccanismi che le potessero alzare e abbassare con il lavoro muscolare, notando che la struttura corporea dell'uomo è meno favorevole, per il volo, di quella degli uccelli.

Sul problema decisivo - quello del profilo alare- Leonardo aveva trovato soluzioni sorprendentemente simili a quelle usate oggi, ma non riuscì a capire che la portanza si genera per scorrimento dell'aria contro la direzione del volo: l'aria scorre più velocemente sulla superficie superiore e meno velocemente su quella inferiore. Un movimento più veloce genera una pressione minore e quindi un movimento verso l'altro. Così si spiega il volo. Eppure Leonardo, studiando l'acqua, si era reso conto che una corrente, incanalandosi in una strettoia, aumenta la sua velocità.

Quello che era mancato a Leonardo era stato il supporto teorico, come egli stesso aveva capito: "Quelli che s'innamoran di pratica senza scienza, son come'l nocchier ch'entra in navilio senza timone o bussola, che mai ha certezza dove si vada" (Ms G., f. 8r).

In Inghilterra, nella contea del Bedfordshire, alcuni appassionati alla storia del volo, guidati da Steve Roberts, si incontrarono con Martin Kemp, celebre studioso di Leonardo. Kemp (1982) mostrò a Roberts e agli altri uno schizzo di Leonardo in cui il rivestimento della superficie portante era avvolto intorno al precedente spigolo della superficie alare, in modo tale da permettere la formazione di un flusso favorevole. Disse Kemp: "Leonardo lo aveva disegnato con molta chiarezza. Dovevamo solo semplificare il suo progetto: invece di costruire due superfici portanti mobili, a destra e a sinistra, costruimmo un'unica parte rigida. Leonardo pensava in modo troppo complicato" (Klein, 2008). Noi riteniamo, però, che il modo di pensare di Leonardo, più che essere complicato, fosse troppo legato alle osservazioni sul volo degli uccelli.

L'aliante di Leonardo, costruito da Kemp e Roberts, fu sperimentato con successo dall'inglese Judy Leden, che aveva vinto tre volte il campionato mondiale di deltaplano.

\section{Visione al di là del mondo: la luna}

Ancora una volta, per le sue osservazioni del cielo, Leonardo si affidò alle sue straordinarie doti visive, capaci di cogliere i più piccoli dettagli tra luci e ombre. Però percepì anche i limiti di queste osservazioni: "Fa occhiali da vedere la luna grande" (CA, f. 518r). L'intepretazione che viene senza dubbio accreditata a Leonardo è quella relativa alla cosiddetta luce cinerea della Luna, cioè il pallido chiarore che s'intravede sulla superficie lunare nella prima fase di Luna crescente, nel cerchio ombroso avvolto dalla sottile falce illuminata dal Sole (Fig. 14). Leonardo spiega correttamente questo fenomeno non come dovuto a luminosità intrinseca della Luna, ma come dovuto al riflesso dei raggi solari sugli oceani della Terra, che giunge a illuminare la parte in ombra della Luna.

"Quando l'occhio vede la Luna in Occidente, vicino al tramontato Sole, esso la vede colla sua parte ombrosa circondata da parte luminosa, 

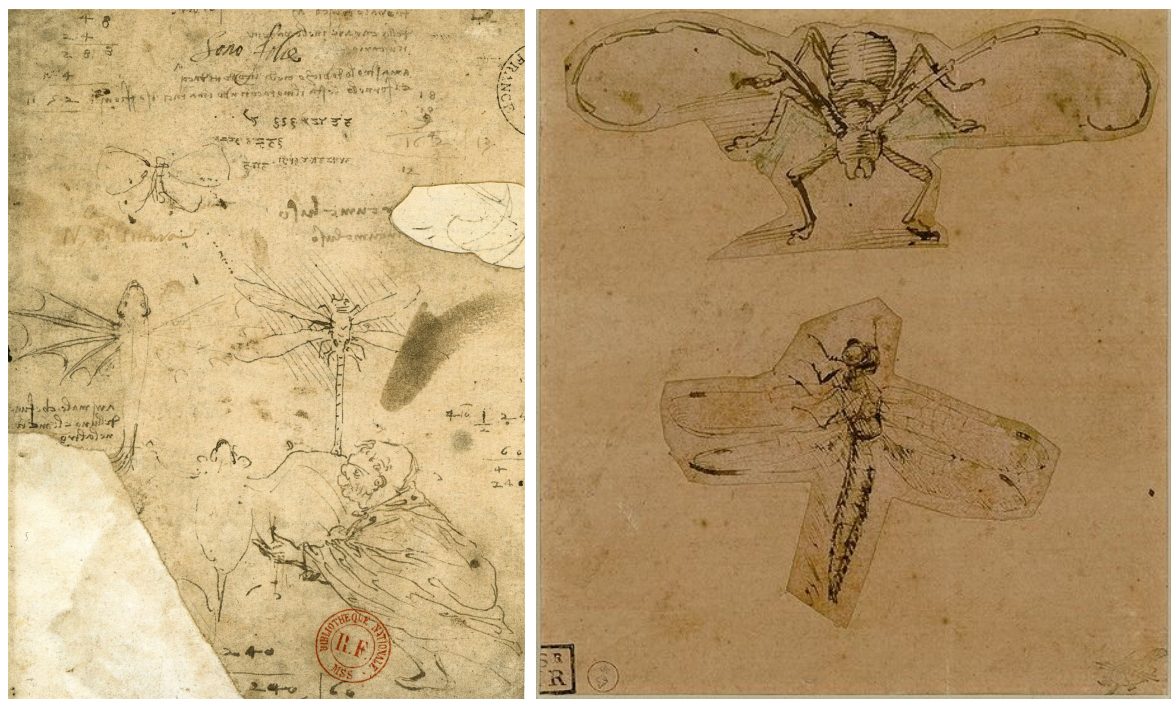

Figura 13: Leonardo, a sinistra: studio di libellula; a destra: studio di insetti (Biblioteca Reale, Torino).

del quale lume la parte laterale e superiore deriva dal Sole, e la parte inferiore deriva dallo oceano occidentale, il quale ancora lui riceve li razzi solari e li rifrette nelle inferiori mari della Luna" (CL, f. 2r).

Il fatto che Leonardo si fidasse solo dei suoi occhi gli permise di distaccarsi dalle credenze astronomiche dei suoi tempi e di formulare ipotesi innovative talvolta corrette e talvolta errate. Egli, ad esempio, credette erroneamente che la Luna fosse circondata, come la Terra, da acqua. Questo perché riteneva che la Luna non avesse una luce propria, e che le sostanze terrose della Luna non potessero riflettere i raggi del Sole, ma questa riflessione fosse possibile solo alle acque del mare. Bisogna immaginare la Luna sfaccettata "come una mora", affinché la sua immagine non sia puntiforme, ma come un disco. L'errata premessa gli permette, però, di rompere con la tradizione aristotelico-tolemaica e di assegnare alla Luna una sua gravità.

"Al presente bisogna provare se essa Luna è corpo grave o lieve: imperò che avendo gravità [...] avessi a discendere inverso il centro dell'universo, e congiungersi con la Terra e, se non per lei, almeno le sue acque avessino a cadere e spogliarla di sé, e cadere in verso il centro e lasciar di sé la Luna spogliata e senza lustro; onde, non seguitando quel che di lei la ragion ci promette, egli è manifesto segno che la Luna è vestita de' sua elementi, cioè acqua, aria e foco, e così in sé sostenga in quello spazio, come fa la nostra terra, co' sua elementi in quest'altro spazio". (Ms K, f. 1r).

\section{Contro la guerra}

Alcuni critici hanno considerato Leonardo un guerrafondaio, in quanto mise il suo ingegno al servizio di condottieri spietati quali Ludovico Sforza di Milano, 


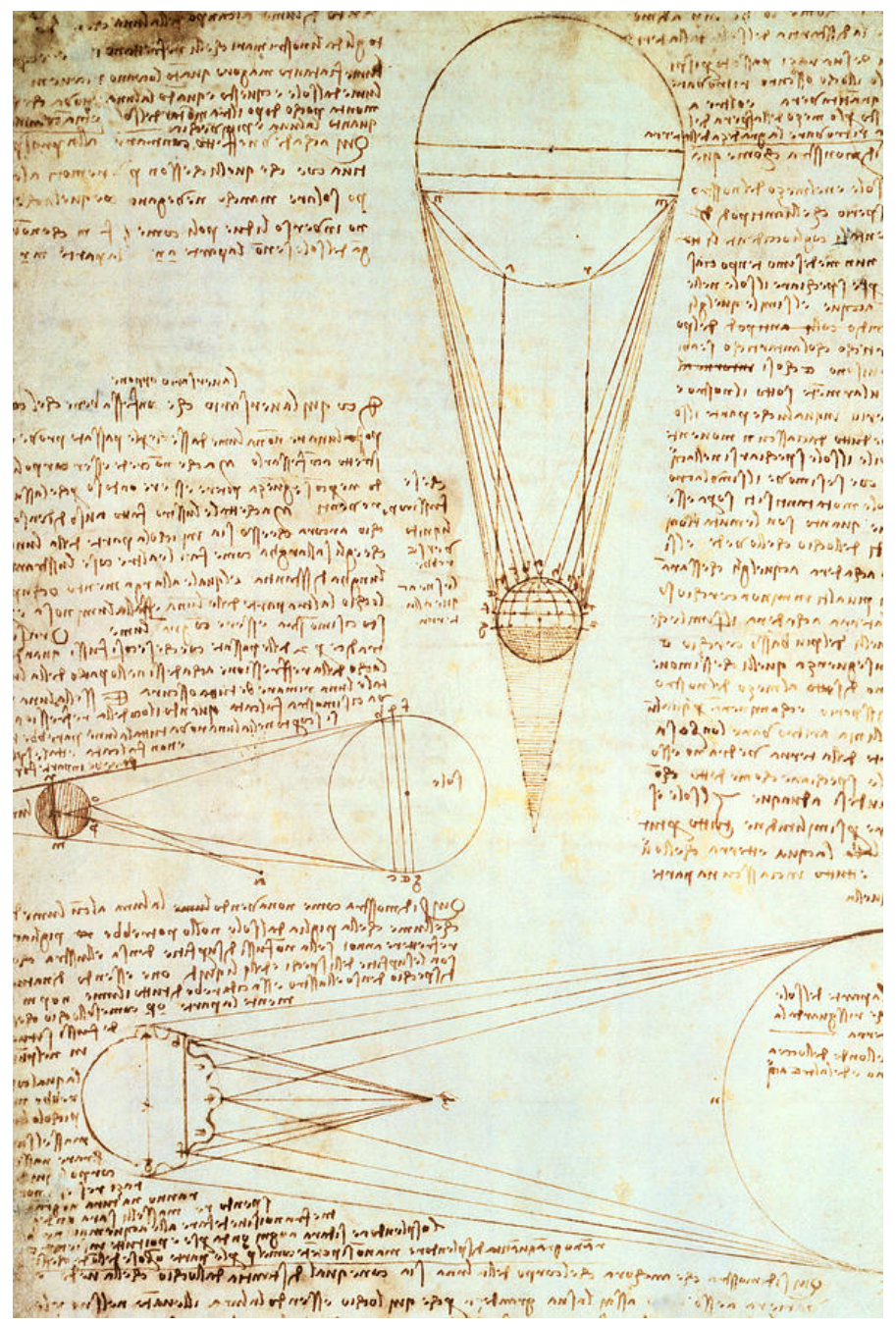

Figura 14: Sole, Terra, Luna e loro rapporti (CL, $f .1 r)$. Nello schizzo in basso si notano le 'onde' che, secondo Leonardo, caratterizzerebbero la superficie lunare.

detto il Moro, e Cesare Borgia (figlio del papa Alessandro VI), detto il Valentino. In effetti Leonardo ideò molte macchine da guerra, come il carro armato, che in gran parte non furono realizzate, ed altri studi bellici. Egli, ad esempio, suggerì la disposizione dei cannoni affinché il bombardamento degli stessi fosse più efficace, e suggerì come allagare certe zone per impedire l'avanzata del nemico, ma sostanzialmente fu contro la guerra. La motivazione di questi studi fu forse simile a quella che spinse Einstein, profondamente pacifista, a scrivere una lettera a favore della costruzione della bomba atomica: la paura dell'avvento di una dittatura disumana. Nei suoi lavori di fisiognomica (lo studio del carattere morale e intellettivo dell'uomo attraverso l'osservazione del suo aspetto somatico), egli ravvisa similarità tra il volto corrucciato di un guerriero e l'immagine della testa di un leone, l'animale feroce per eccellenza (Fig. 15).

"Quello che caratterizza, infatti, gli uomini bestiali è uno scarso uso della ragione ed il lasciarsi dominare dalle passioni. Negli uomini che si abbandonano frequentemente all'ira, la parte passionale ed 


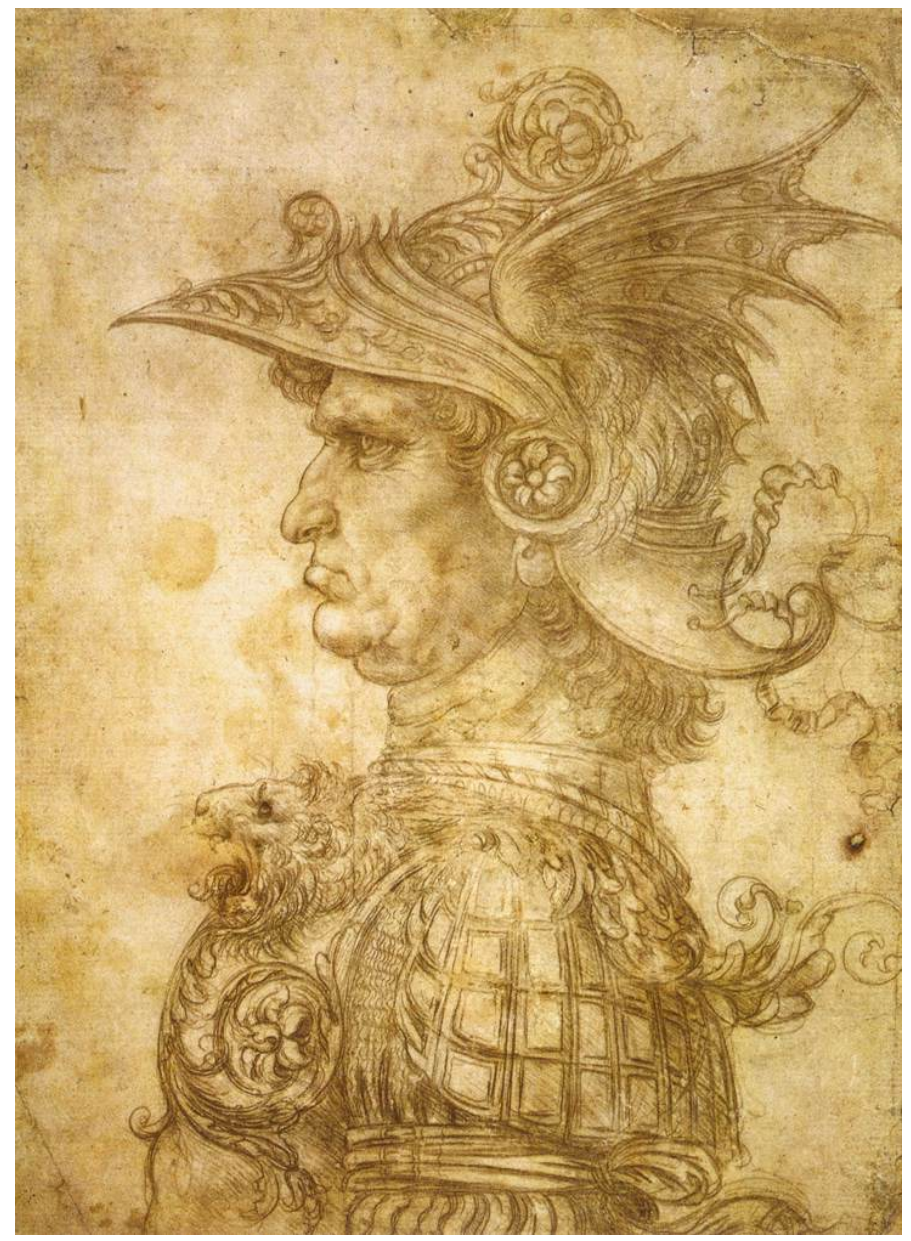

Figura 15: Leonardo, Testa di guerriero (British Museum, Londra).

animale della sua anima è molto forte, a scapito di quella razionale ed umana."

Inoltre, ebbe a definire la guerra "pazzia, bestialissima" (LDP 177). Questa convinzione emerge chiaramente nella Battaglia d'Anghiari. Questo grande dipinto gli era stato commissionato per il Salone dei Cinquecento, in Palazzo Vecchio a Firenze, per celebrare la vittoria di Firenze su Milano (29 giugno 1440); ma volendo Leonardo sperimentare una nuova tecnica che gli permettesse di dipingere con più calma, prima che l'intonaco si asciugasse, il dipinto andò presto perduto. Di esso, però, restano alcuni bozzetti dello stesso Leonardo, ed alcune copie, delle quali è particolarmente pregevole quella dovuta a Rubens (Fig. 16).

In questo dipinto la testa di un cavallo è sostituita dalla testa di un guerriero, che è diventato una bestia. Il messaggio di Leonardo è chiarissimo: la guerra trasforma l'uomo in bestia.

\subsection{L'alchimia e l'astrologia}

Tra il XIII e il XVI secolo la convinzione che l'uomo sia fatto ad immagine di Dio portò ad attenuare l'analogia tra uomo e animale, e a sviluppare la parte 'di- 


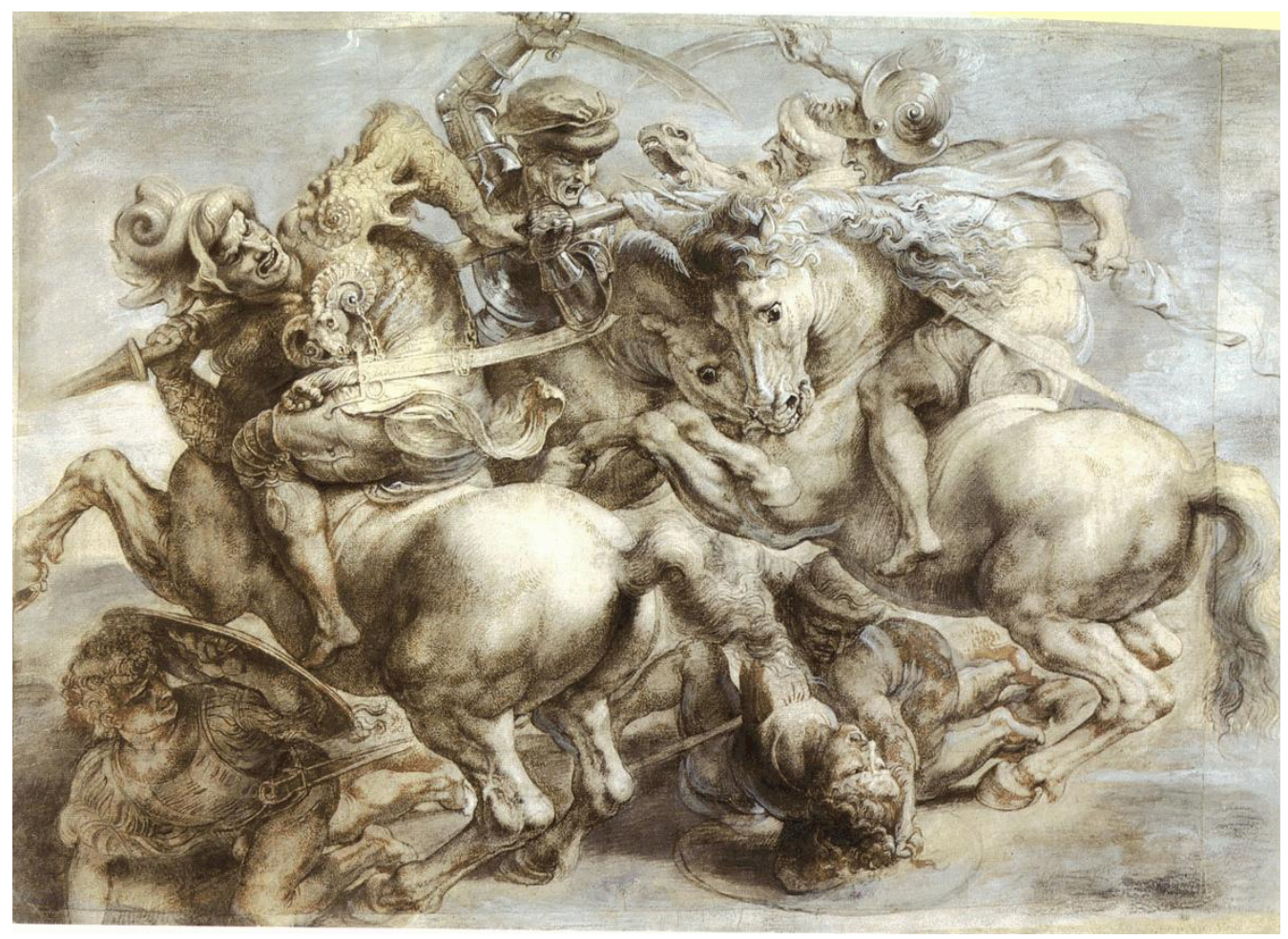

Figura 16: Battaglia di Anghiari, bozzetto di Peter Paul Rubens (1577-1640; Louvre, Parigi).

vina' o 'celeste' dell'essere umano. Questo portò all'astro-biologia o fisiognomica astrologica. Si sviluppò cioè una 'scienza' che cercava di interpretare i fenomeni biologici anche in termini degli influssi celesti. La chiromanzia, cioè la possibilità di divinare il futuro di un individuo partendo dai segni della sua mano, divennero oggetto di approfonditi studi 'scientifici'. Sia i filosofi naturali che i medici presero in seria considerazione l'influenza degli astri sullo sviluppo dell'embrione. Leonardo, che si affidò solo ai risultati delle dissezioni dei cadaveri, manifestò una chiusura totale nei confronti dell'astrologia. Meno drastico fu nei confronti dell'alchimia (la chimica dei suoi tempi), che definì l'arte di formare composti partendo da alcuni elementi. Mise però in guardia i suoi simili, perché le sostanze ottenute erano spesso "velenose e nocive all'uomo".

\section{L'eredità di Leonardo}

I circa diecimila fogli lasciati da Leonardo al discepolo Melzi, dopo la morte di questi, andarono in parte perduti ed in parte sparsi tra numerose biblioteche o acquisiti da privati. Oggi restano circa 6000 fogli. L'influenza dei suoi disegni sulle future invenzioni, anche se spesso non riconosciuta, è stata grandissima. Chi ha dato uno sguardo ai suoi schizzi ha potuto progettare un futuro fatto di macchine volanti, terribili catapulte, elicotteri, macchine tessili, automi in figura umana, scafandri di palombari, robot, canali di navigazione ed irrigazione, montagne forate da gallerie, splendide cattedrali e città ideali ... Lo spirito scientifico 


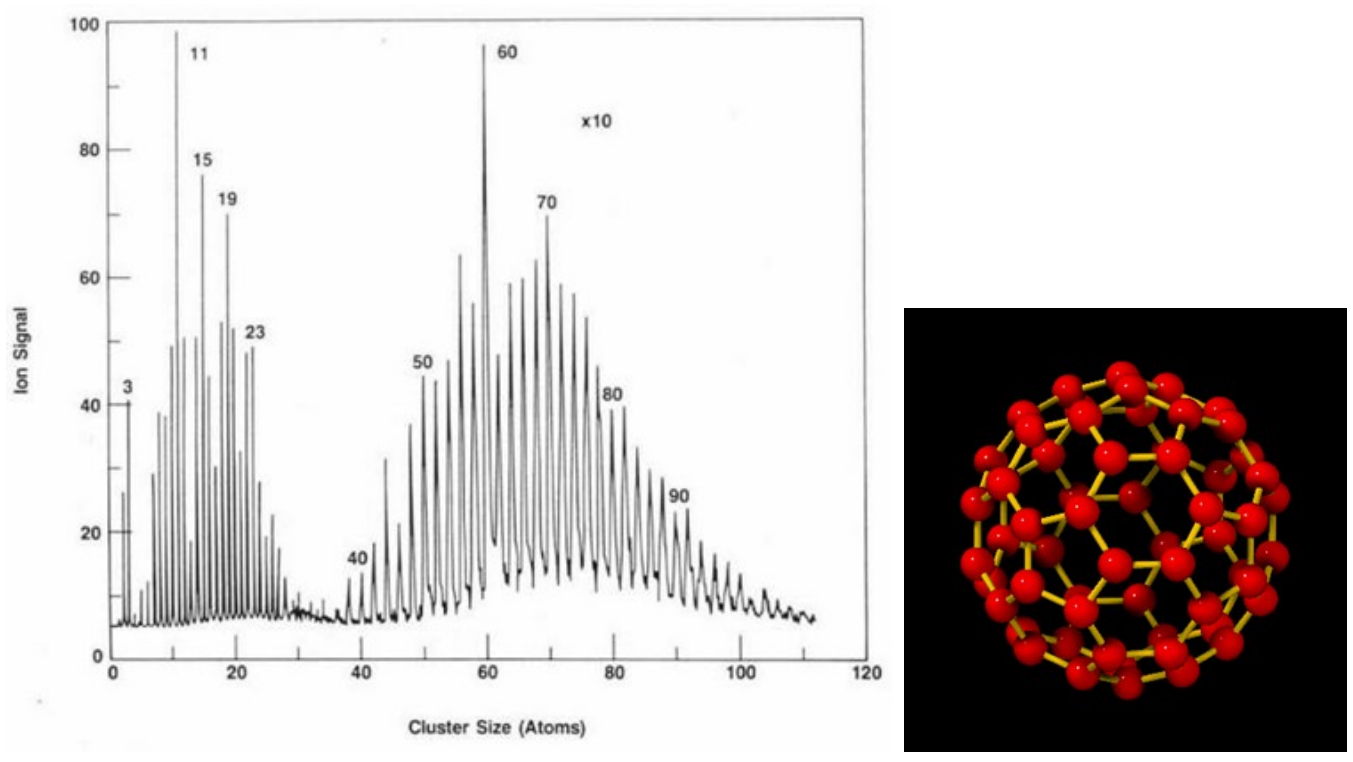

Figura 17: I risultati di Kroto et al. (1985) sui fullereni.

di Leonardo si sviluppò per la sua capacità di interessarsi e meravigliarsi di tutto, di porsi domande sui più disparati settori della natura, di considerare il mondo un'unità e di cercare quindi somiglianze fra i fenomeni più lontani. La vera eredità di Leonardo è l'aver mostrato di cosa è capace l'uomo quando si libera dalle costrizioni e dalle apparenti certezze delle idee dominanti, nel periodo in cui vive.

\subsection{Un'inattesa attualità: i fullereni}

Studiando le polveri interstellari (ricche di atomi di carbonio), attorno agli anni '80, alcuni scienziati osservarono delle linee di assorbimento simili alle gobbe di un cammello, appartenenti ad una molecola sconosciuta. Kroto e Smalley iniziarono quindi una serie di esperimenti di laser ablation di grafite in ambiente saturo di elio. Tali studi culminarono nel loro lavoro su Nature (Kroto et al., 1985) in cui fecero vedere che lo spettro in massa dei cluster da loro ottenuti mostrava un picco prominente per una molecola contenente 60 atomi di carbonio

Kroto e Smalley interpretarono questo risultato, che valse loro il Premio Nobel per la Chimica, insieme a Curl, nel 1996, come dovuto ad una molecola che aveva la forma di un icosaedro troncato, costituito cioè da 12 pentagoni e 20 esagoni, l'unica figura che permetteva che ogni atomo di carbonio fosse legato ad altri tre atomi, dando quindi alla molecola una stabilità relativamente elevata. Inizialmente questa molecola fu chiamata soccerball (pallone da calcio), ma questo nome sembrò in seguito troppo banale, e fu quindi chiamata buckminsterfullerene (dal nome di Buckminster-Fuller, architetto, che aveva progettato cupole di questa forma). Successivamente, il nome fu abbreviato in fullerene.

Il primo convegno scientifico sui fullereni che si tenne in Italia (a Bologna, nel 1992; Fig. 18) fece notare che Leonardo era stato uno dei primi a disegnare questa forma geometrica. Inoltre lo stesso Buckminster-Fuller aveva ammesso 


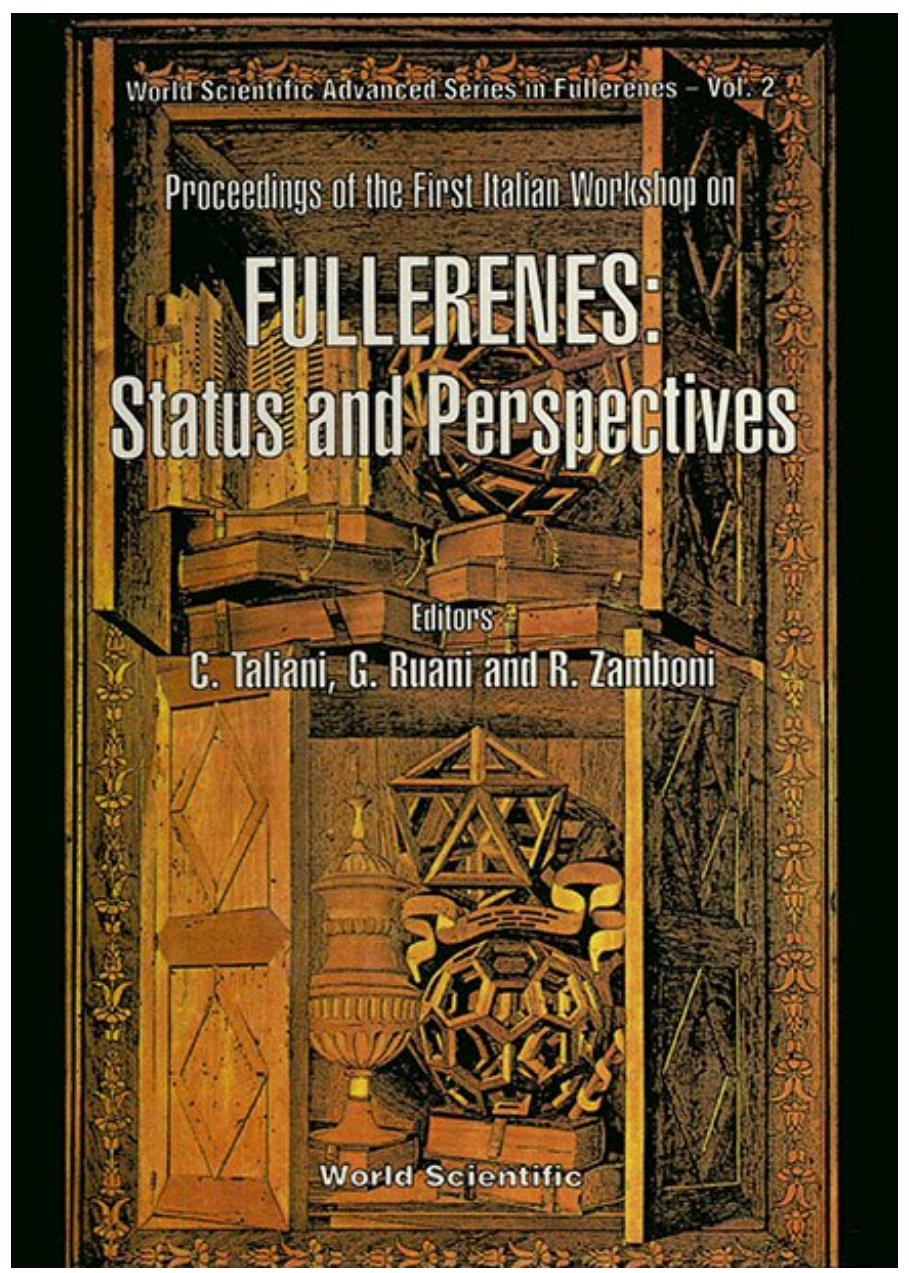

Figura 18: La copertina di Taliani et al. (1992).

di essersi ispirato ai disegni di Leonardo (Pedretti, 2008). Questa molecola si sarebbe dovuta chiamare Leonardium, ma ormai era troppo tardi ...

\subsection{La sezione aurea e la topologia}

Leonardo era un fanatico delle simmetrie e delle proporzioni, ed il numero che governa l'armonia della natura (oltre $\pi$ ) è la sezione aurea

$$
\phi=\frac{1+\sqrt{5}}{2}=1.618 \ldots
$$

Tutto il nostro corpo, come descritto da Leonardo, rispetta la sezione aurea. Ad esempio nella nostra mano, il rapporto fra le lunghezze medie di falangina e falange è uguale a $\phi$. La sezione aurea si manifesta in vari modi: nel DNA, nelle galassie, nella conchiglia del nautilus, nella serie di Fibonacci, nella spirale logaritmica, negli angoli tra le foglie di un fiore, nella proporzione fra i lati delle tessere magnetiche come le carte di credito ...

Inoltre, Leonardo dedicò decine di fogli dei suoi codici per studiare le trasformazioni continue di figure piane e solidi rettilinei (cilindri, piramidi etc) in 


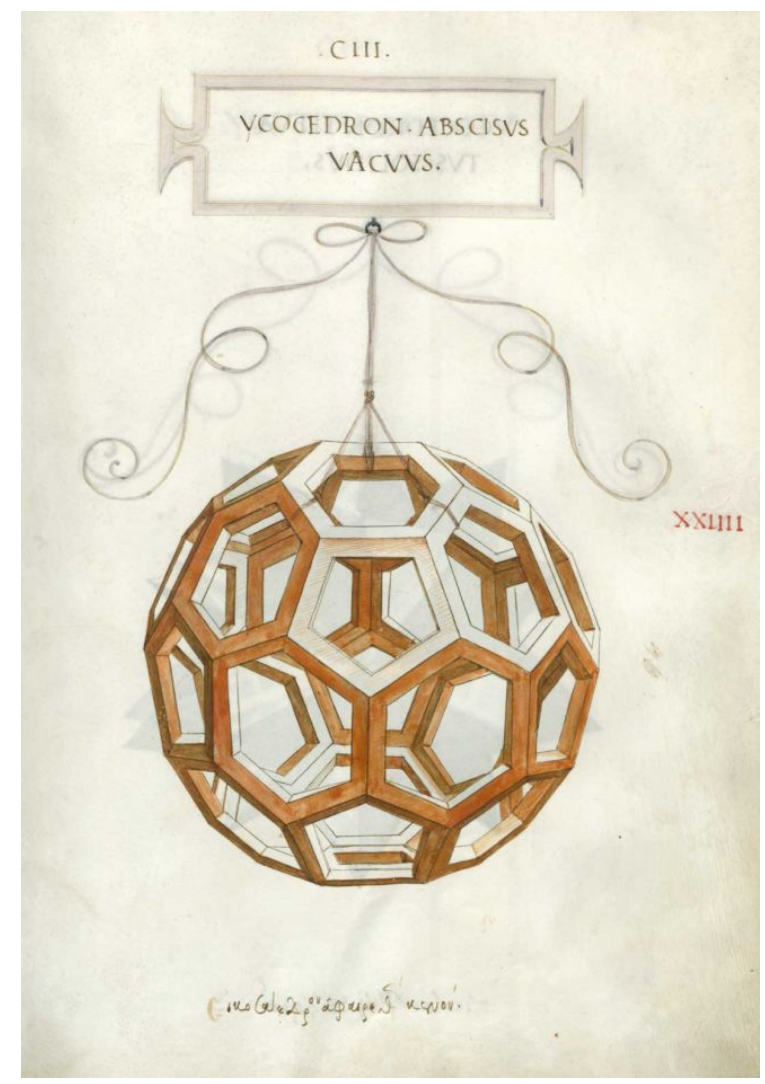

Figura 19: Ancora da Pacioli (1497): icosaedro troncato.

oggetti curvilinei, in maniera tale che le aree e i volumi si conservassero. Egli considera poligoni inscritti in un cerchio e 'ripiega' le facce che stanno tra i lati del poligono e la circonferenza in modo da ottenere stelle curvilinee di grande complessità ed armonia. Costruisce figure, disciplinate da una regola ripetitiva che serve a "variare in infinito" una o più superfici, mantenendone immutata la quantità.

A differenza delle geometria euclidea, che riguardava figure rigide e forme statiche, la geometria di Leonardo è una geometria dinamica, una geometria delle deformazioni continue (Fiscaletti, 2016). Egli, oltre a usare la geometria per studiare la traiettoria dei corpi, usa il movimento per dimostrare teoremi geometrici. Egli definisce questo modo di procedere "geometria che si prova col moto" o che "si fa col moto" (CODICE DI MADRID, II, F. 107R). Esaminando i disegni di Leonardo, si può dedurre che le trasformazioni da lui condotte delle figure geometriche sono trasformazioni topologiche, cioè trasformazioni per cui le proprietà della figura in considerazione rimangono invariate quando la figura viene piegata, stirata, compressa o deformata in qualsiasi modo che non crei nuovi punti o ne fonda insieme altri (Fiscaletti). Giustamente viene attribuita a Leibniz ed a Poincaré la fondazione della topologia, ma senza dubbio Leonardo con la sua "geometria che si fa col moto" fornì delle anticipazioni a questa straordinaria ed attuale branca della matematica. 


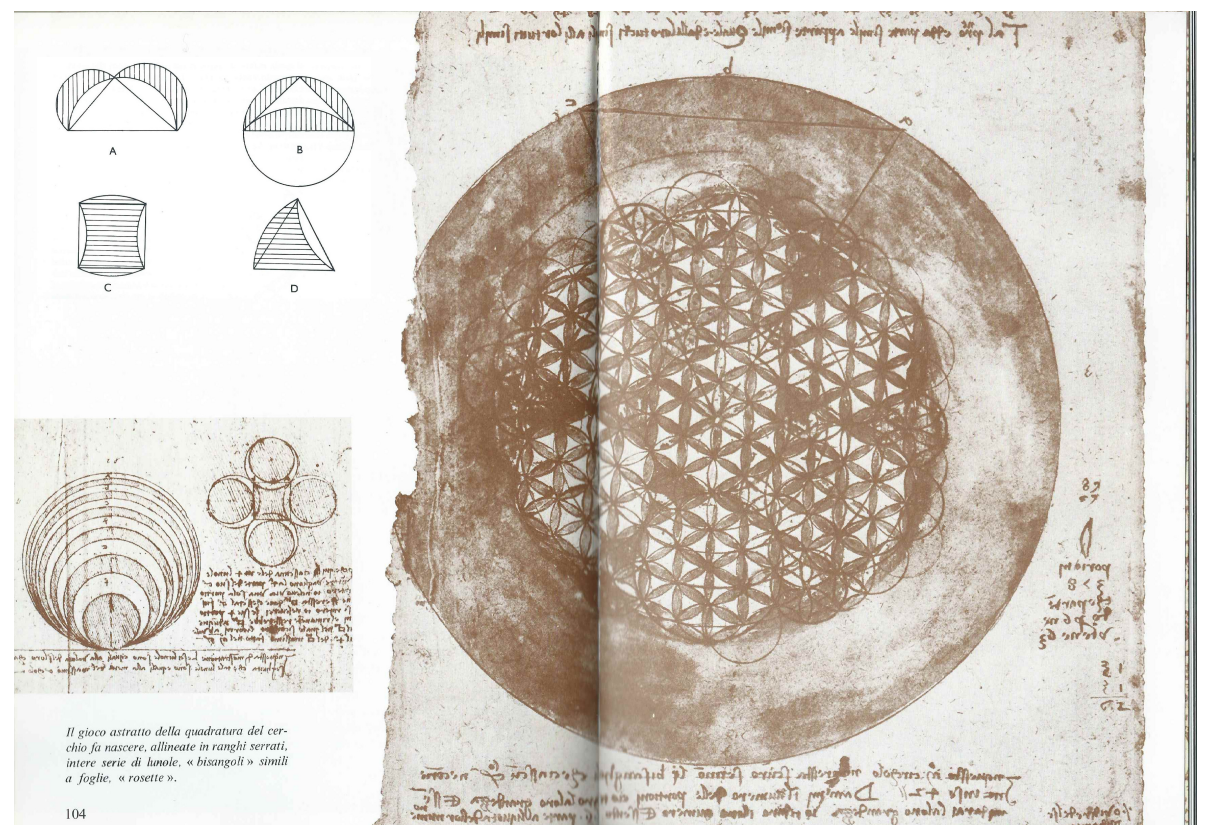

Figura 20: Lunule.

\section{Abbreviazioni}

CA: Codex Atlanticus (Biblioteca Ambrosiana: Milano)

CL: Codex Leicester (precedentemente Codex Hammer, oggi fa parte della collezione privata di Bill Gates)

CV: Codice sul volo degli uccelli (Biblioteca Reale: Torino)

Ms E: Manoscritto E di Francia (Institut de France: Parigi)

Ms G: Manoscritto G di Francia (Institut de France: Parigi)

Ms I: Manoscritto I di Francia (Institut de France: Parigi)

Ms K: Manoscritto K di Francia (Institut de France: Parigi)

RC: The Royal Collection (Windsor Castle: Berkshire, UK)

\section{Bibliografia}

Anderson, P. W. (1972). More Is Different. Science 177(4047), 393. doi:10.1126/science.177.4047.393.

Barras, C. (2012). Leonardo fossil sketch may depict early nests. Nature doi:10.1038/nature.2012.11841.

Buridanus, I. (1509). Quaestiones super tres libros De coelo et mundo. In Subtilissimae Quaestiones super octo Physicorum libros Aristotelis (Parigi). 
Canguilhem, G. (1968). Etudes d'histoire et de philosophie des sciences (Vrin, Paris). Traduzione italiana Introduzione alla storia delle scienze (Milano: Jaca Books, 1973).

Dyson, F. J. (2006). The Scientist as Rebel (New York Review Books, New York).

Feynman, R. P. (1949). Space-Time Approach to Quantum Electrodynamics. Phys. Rev. 76, 769. doi:10.1103/PhysRev.76.769.

Fiscaletti, D. (2016). Dalla matematica di Leonardo alla teoria della complessità: la visione dinamica della geometria. Scienza e conoscenza 58, 10.

Galluzzi, P., editor (2018). L'acqua microscopio della natura: il Codice Leicester di Leonardo da Vinci (Giunti, Firenze).

Kaiser, D. (2005). Drawing Theories Apart. The Dispersion of Feynman Diagrams in Postwar Physics (The University of Chicago Press, Chicago).

Kemp, M. (1982). Leonardo da Vinci: le mirabili operazioni della natura e dell'uomo (Mondadori, Milano).

Klein, S. (2008). Da Vinci Vermächtnis, oder, Wie Leonardo die Welt neu erfand (S. Fischer Verlag, Frankfurt). Traduzione italiana: L'eredità di Leonardo. Il genio che reinventò il mondo, (Torino: Bollati Boringhieri, 2017).

Kroto, H. W., Heath, J. R., O’Brien, S. C., Curl, R. F., and Smalley, R. E. (1985). $C_{60}$ : Buckminsterfullerene. Nature 318(6042), 162. doi:10.1038/318162a0.

Kuhn, T. S. (1962). The Structure of Scientific Revolutions (University of Chicago Press, Chicago).

Laurenza, D. (1999). Leonardo: la scienza trasfigurata in arte, volume 9 of I grandi della scienza (Le Scienze, Roma).

Maccarrone, D. (2019). Comunicazione privata.

Nabokov, V. (1966). Speak, memory : an autobiography revisited (Putnam, New York). Traduzione italiana: Parla, ricordo. Un'autobiografia rivisitata (Milano: Adelphi, 2010).

Pacioli, L. (1497). De Divina Proportione (Paganino Paganini, Venezia).

Pedretti, C. (2008). Leonardo E io (Mondadori, Milano).

Popper, K. R. (1935). Logik der Forschung: zur Erkenntnistheorie der modernen Naturwissenschaft (Springer, Wien).

Popper, K. R. (1963). Conjectures and refutations (Routledge, London).

Pucci, R. (2017). Symmetries and physics. Opening lecture (November 30, 2002) of the Academic Year 2002-2003 of the University of Catania. In Angilella, G. G. N. and La Magna, A., editors, Correlations in condensed matter under extreme conditions: a tribute to Renato Pucci on the occasion of his 70th birthday, chapter 25, pages 345357 (Springer, New York, Berlin, Heidelberg). doi:10.1007/978-3-319-53664425.

Pucci, R. and Angilella, G. G. N. (2017). The bold and the humble: physics and epistemology. In Angilella, G. G. N. and La Magna, A., editors, Correlations in condensed matter under extreme conditions: a tribute to Renato Pucci on the occasion of his 70th birthday, chapter 27, pages 369-372 (Springer, New York, Berlin, Heidelberg). doi:10.1007/978-3-319-53664-4_27.

Rovelli, C. (2011). The first scientist. Anaximander and his legacy (Westholme, Yardley). 
Rovelli, C. (2015). Lolita e l'icaro azzurro. Domenica de Il Sole 24 Ore Reprinted in Rovelli (2018).

Rovelli, C. (2018). Ci sono luoghi al mondo dove più che le regole è importante la gentilezza (Corriere della Sera, Milano).

Taliani, C., Ruani, G., and Zamboni, R., editors (1992). Proceedings of the first Italian workshop on Fullerenes: status and perspectives, volume 2 of World Scientific advanced series in fullerenes (World Scientific, Singapore).

Treccani (2015). Leonardo. In Enciclopedia Treccani (Istituto della Enciclopedia Italiana, Roma).

Vasari, G. (1568). Le vite de' più eccellenti pittori, scultori, e architettori (Giunti, Firenze), second edition.

Wilczek, F. (2015). A beautiful question (Penguin, New York). 